\title{
Revisiting a generalized two-Higgs-doublet model in light of the muon anomaly and lepton flavor violating decays at the HL-LHC
}

\author{
Nivedita Ghosh $\odot^{*}$ and Jayita Lahiri ${ }^{\dagger}$ \\ Regional Centre for Accelerator-based Particle Physics, Harish-Chandra Research Institute, \\ HBNI, Chhatnag Road, Jhunsi, Allahabad-211 019, India
}

(Received 28 October 2020; accepted 25 January 2021; published 16 March 2021)

\begin{abstract}
One of the main motivations to look beyond the Standard Model is the discrepancy between the theoretical prediction and observation of the anomalous magnetic moment of the muon. Alleviating this tension between theory and experiment and simultaneously satisfying the bounds from lepton flavor violation data is a challenging task. In this paper, we consider a generalized two-Higgs-doublet model with a Yukawa structure as a perturbation of a type $\mathrm{X}$ two-Higgs-doublet model. In view of this model, we explore the muon anomaly and lepton flavor violation along with constraints coming from $B$ physics, theoretical constraints, electroweak observables, and collider data which can restrict the model parameter space significantly. We find that within the framework of this model it is possible to obtain regions allowed by all constraints that can provide an explanation for the observed muon anomaly and at the same time predict interesting signatures of lepton flavor violation. Furthermore, we consider the flavor-violating decay of a low-mass $C P$-odd scalar to probe the allowed parameter space at future runs of the LHC. With a simple cut-based analysis, we show that part of this parameter space can be probed with a significance $>5 \sigma$. We also provide an artificial neural network analysis that significantly improves our cut-based results.
\end{abstract}

DOI: 10.1103/PhysRevD.103.055009

\section{INTRODUCTION}

A $125 \mathrm{GeV}$ scalar, with a striking resemblance to the Higgs boson proposed in the Standard Model (SM), was observed at the LHC on July 4, 2012 [1,2]. Experimental evidence of its increasing bias towards the SM puts stringent limits on new physics (NP) scenarios. However, there are still many unanswered questions that indicate that the SM is not a complete theory and it is imperative to go beyond it. The anomalous magnetic moment of the muon is one such crucial observation that calls for new physics. There is a long-standing discrepancy between the SM prediction for the magnetic moment of the muon and its observed experimental value [3]. The ongoing E989 experiment at Fermilab [4] and future E34 experiment at J-PARC [5] are expected to shed new light on this tension between theory and data.

On the other hand, though lepton flavor violation (LFV) has been observed in neutrino oscillation experiments [6,7], LFV has not yet been observed in the charged lepton sector.

\footnotetext{
niveditaghosh@hri.res.in

jayitalahiri@hri.res.in
}

Published by the American Physical Society under the terms of the Creative Commons Attribution 4.0 International license. Further distribution of this work must maintain attribution to the author(s) and the published article's title, journal citation, and DOI. Funded by SCOAP ${ }^{3}$.
Various low-energy experiments [8-15] have put strong bounds on the branching ratios of flavor-changing neutral current (FCNC) decays and correspondingly on the associated couplings.

The two phenomena described above-namely, the muon anomaly and lepton flavor violation - are intricately connected with each other. Experimental observation of the muon anomaly and the nonobservation of lepton flavor violation will definitely create tension in terms of the allowed parameter space for various candidate models that satisfy these two results individually. Models that can accommodate the muon anomaly in general face severe LFV constraints $[13,16]$. In this work, our goal is to satisfy both of these observations simultaneously and look for signatures of lepton flavor violation in collider experiments, which is an independent search strategy altogether [17-20]. If in the future LFV is observed in low-energy experiments, a simultaneous observation of LFV processes in collider experiments will be a telltale signature. On the other hand, the nonobservation of LFV processes at in collider experiments will help to constrain the model parameter space.

In this work we consider a minimal scalar extension of the SM, i.e., a generalized two-Higgs-doublet model (2HDM) [21-23], with a Yukawa structure as a perturbation of a type $\mathrm{X} 2 \mathrm{HDM}$. The presence of nonstandard light scalars in the 2HDM allows one to satisfy the muon anomaly, some examples of which can be found in the 
literature [24-41]. The theory and phenomenology of FCNCs in the Yukawa sector of 2HDMs has also been studied in detail in the literature [42-47]. However, studies of $2 \mathrm{HDMs}$ in light of both the muon anomaly and LFV are scarce in the literature $[48,49]$.

Apart from finding a suitable region of parameter space where both the muon anomaly and LFV constraints are satisfied at two loops, along with theoretical constraints coming from perturbativity, unitarity, vacuum stability, oblique parameter constraints and constraints coming from $B$ physics and collider experiments, we look for a flavorviolating decay of the $C P$-odd scalar in the $\ell^{+} \ell^{\prime-}+\mathbb{E}_{T}$ final state, where $\ell, \ell^{\prime}=e, \mu$. This channel comes from the flavor-violating decay of the $C P$-odd Higgs, $A \rightarrow \ell \tau_{\ell^{\prime}}$, where $\tau_{\ell^{\prime}}$ implies $\tau$ decaying leptonically. With a simple cut-based analysis we show that a selected region of parameter space can be probed at the future highluminosity collider HL-LHC. We also perform an artificial neural network (ANN) analysis and see that, compared to the cut-based analysis, this parameter space can be probed with even lower luminosity.

Our work is organized as follows. In Sec. II we present a brief outline of the model. In Sec. III we discuss the muon anomaly and its impact on our model parameter space. We then move to Sec. IV where we explore the allowed parameter space, taking into account the theoretical constraints, and constraints coming from low-energy observables and collider experiments. We present both a cut-based and a neural-network-based collider analysis in Sec. V. We summarize our results and conclude in Sec. VI.

\section{THE GENERALIZED TWO-HIGGS-DOUBLET MODEL}

In this section we briefly discuss the model in consideration. We follow the convention of Ref. [18]. ${ }^{1}$ Two scalar doublets $\Phi_{1}$ and $\Phi_{2}$ with hypercharge $Y=1$ are present in this model. ${ }^{2}$ The most general scalar potential can be written as

$$
\begin{aligned}
\mathcal{V}_{2 \mathrm{HDM}}= & m_{11}^{2}\left(\Phi_{1}^{\dagger} \Phi_{1}\right)+m_{22}^{2}\left(\Phi_{2}^{\dagger} \Phi_{2}\right)-\left[m_{12}^{2}\left(\Phi_{1}^{\dagger} \Phi_{2}\right)+\text { H.c. }\right] \\
& +\frac{1}{2} \lambda_{1}\left(\Phi_{1}^{\dagger} \Phi_{1}\right)^{2}+\frac{1}{2} \lambda_{2}\left(\Phi_{2}^{\dagger} \Phi_{2}\right)^{2} \\
& +\lambda_{3}\left(\Phi_{1}^{\dagger} \Phi_{1}\right)\left(\Phi_{2}^{\dagger} \Phi_{2}\right)+\lambda_{4}\left(\Phi_{1}^{\dagger} \Phi_{2}\right)\left(\Phi_{2}^{\dagger} \Phi_{1}\right) \\
& +\left\{\frac{1}{2} \lambda_{5}\left(\Phi_{1}^{\dagger} \Phi_{2}\right)^{2}+\left[\lambda_{6}\left(\Phi_{1}^{\dagger} \Phi_{1}\right)+\lambda_{7}\left(\Phi_{2}^{\dagger} \Phi_{2}\right)\right]\right. \\
& \left.\times\left(\Phi_{1}^{\dagger} \Phi_{2}\right)+\text { H.c. }\right\}
\end{aligned}
$$

where H.c. denotes the Hermitian-conjugate term.

\footnotetext{
${ }^{1}$ For a general 2HDM review, see Ref. [50].

${ }^{2} Q=T_{3}+\frac{Y}{2}$.
}

In general, $m_{12}^{2}, \lambda_{5}, \lambda_{6}$, and $\lambda_{7}$ can be complex, while the rest of the parameters are real. However, in this work we assume that $C P$ is conserved, and therefore $m_{12}^{2}, \lambda_{5}, \lambda_{6}$, and $\lambda_{7}$ are taken to be real. We should mention here that in the absence of $Z_{2}$ symmetry $\left(\Phi_{1} \rightarrow \Phi_{1}, \Phi_{2} \rightarrow-\Phi_{2}\right) \lambda_{6}$ and $\lambda_{7}$ can take nonzero values in general. ${ }^{3}$

The two scalar doublets of the model can be expanded as

$$
\begin{aligned}
& \Phi_{1}=\left(\begin{array}{c}
\phi_{1}^{+} \\
\frac{1}{\sqrt{2}}\left(\operatorname{Re}\left[\Phi_{1}^{0}\right]+i \operatorname{Im}\left[\Phi_{1}^{0}\right]\right)
\end{array}\right), \\
& \Phi_{2}=\left(\begin{array}{c}
\phi_{2}^{+} \\
\frac{1}{\sqrt{2}}\left(\operatorname{Re}\left[\Phi_{2}^{0}\right]+i \operatorname{Im}\left[\Phi_{2}^{0}\right]\right)
\end{array}\right) .
\end{aligned}
$$

After electroweak symmetry breaking the doublets acquire vacuum expectation values (VEVs),

$$
\left\langle\Phi_{1}\right\rangle=\frac{1}{\sqrt{2}}\left(\begin{array}{c}
0 \\
v_{1}
\end{array}\right), \quad\left\langle\Phi_{2}\right\rangle=\frac{1}{\sqrt{2}}\left(\begin{array}{c}
0 \\
v_{2}
\end{array}\right) .
$$

A key parameter of the model is $\tan \beta=\frac{v_{2}}{v_{1}}$. Charged Goldstones $\left(G^{ \pm}\right)$and physical charged Higgs states $\left(H^{ \pm}\right)$ are produced as a linear combination of $\phi_{1}^{ \pm}$and $\phi_{2}^{ \pm}$. On the other hand, the same linear combination of $\operatorname{Im}\left[\Phi_{1}^{0}\right]$ and $\operatorname{Im}\left[\Phi_{2}^{0}\right]$ gives rise to a neutral $C P$-odd Goldstone $\left(G^{0}\right)$ and a physical $C P$-odd state $(A)$. The mixing is shown in the following equations:

$$
\begin{gathered}
\left(\begin{array}{c}
\phi_{1}^{ \pm} \\
\phi_{2}^{ \pm}
\end{array}\right)=\left(\begin{array}{cc}
\cos \beta & \sin \beta \\
-\sin \beta & \cos \beta
\end{array}\right)\left(\begin{array}{c}
H^{ \pm} \\
G^{ \pm}
\end{array}\right), \\
\left(\begin{array}{c}
\sqrt{2} \operatorname{Im}\left[\Phi_{1}^{0}\right] \\
\sqrt{2} \operatorname{Im}\left[\Phi_{2}^{0}\right]
\end{array}\right)=\left(\begin{array}{cc}
\cos \beta & \sin \beta \\
-\sin \beta & \cos \beta
\end{array}\right)\left(\begin{array}{c}
A \\
G^{0}
\end{array}\right) .
\end{gathered}
$$

Diagonalizing the mass matrix for the $C P$-even neutral states, we get the mass eigenstates $h$ and $H$, where the states in the mass basis and flavor basis are related by the following rotation:

$$
\left(\begin{array}{c}
\sqrt{2} \operatorname{Re}\left[\Phi_{1}^{0}\right]-v_{1} \\
\sqrt{2} \operatorname{Re}\left[\Phi_{2}^{0}\right]-v_{2}
\end{array}\right)=\left(\begin{array}{cc}
\cos \alpha & \sin \alpha \\
-\sin \alpha & \cos \alpha
\end{array}\right)\left(\begin{array}{c}
h \\
H
\end{array}\right),
$$

where either $h$ or $H$ is assumed to behave like the SM Higgs with mass $125 \mathrm{GeV}$.

Having discussed the Higgs potential of a generalized $2 \mathrm{HDM}$, we proceed to the Yukawa sector of the model. It is well known that to avoid tree-level flavor-changing neutral currents, a $Z_{2}$ symmetry is imposed on the general Yukawa

\footnotetext{
${ }^{3}$ Throughout the paper we vary $\lambda_{6}$ and $\lambda_{7}$ between 0 to 0.1 .
} 
Lagrangian of the 2HDM. The doublets $\Phi_{1}$ and $\Phi_{2}$ and the fermion fields behave as either even or odd under this $Z_{2}$ symmetry, and depending on this behavior four common types of 2HDM are formed: type I, type II, lepton-specific or type X, and flipped or type Y. In type I, up- and downtype quarks and leptons couple to $\Phi_{2}$. In type II, up-type quarks couple to $\Phi_{2}$ and down-type quarks and leptons couple to $\Phi_{1}$. In type X, up- and down-type quarks couple to $\Phi_{2}$ and leptons couple to $\Phi_{1}$. In type Y, up-type quarks and leptons couple to $\Phi_{2}$ and the down-type quarks couple to $\Phi_{1}$.

Unlike these aforementioned types of $2 \mathrm{HDM}$, in the generalized $2 \mathrm{HDM}$ no $Z_{2}$ symmetry is imposed on the Lagrangian and therefore this model produces tree-level FCNCs. In this case both doublets $\Phi_{1}$ and $\Phi_{2}$ couple to all of the leptons and quarks. In the generalized 2HDM, the Yukawa couplings to the quarks and leptons can be written as

$$
\begin{aligned}
-\mathcal{L}_{\text {Yukawa }}= & \bar{Q}_{L}\left(Y_{1}^{d} \Phi_{1}+Y_{2}^{d} \Phi_{2}\right) d_{R}+\bar{Q}_{L}\left(Y_{1}^{u} \tilde{\Phi}_{1}+Y_{2}^{u} \tilde{\Phi}_{2}\right) u_{R} \\
& +\bar{L}_{L}\left(Y_{1}^{\ell} \Phi_{1}+Y_{2}^{\ell} \Phi_{2}\right) e_{R}+\text { H.c. }
\end{aligned}
$$

In Eq. (7), $Y_{1,2}^{u, d, \ell}$ are the Yukawa matrices (whose flavor indices have been suppressed) and $\tilde{\Phi}_{i}=i \sigma_{2} \Phi_{i}^{*}$. Expanding this equation in terms of the VEVs and physical fields, we get the fermion mass matrix

$$
\bar{f}_{L} \mathbf{M}^{f} f_{R}=\bar{f}_{L}\left(\frac{v_{1} Y_{1}^{f}}{\sqrt{2}}+\frac{v_{2} Y_{2}^{f}}{\sqrt{2}}\right) f_{R}+\text { H.c. }
$$

Without assuming any particular relation between the matrices $Y_{1}$ and $Y_{2}$, it is not possible to diagonalize the two of them simultaneously, which leads to tree-level scalar-mediated FCNCs. To diagonalize the fermion mass matrices by biunitary transformation we need two unitary matrices $U_{L}^{f}$ and $U_{R}^{f}$. We adopt the convention of Ref. [51] and consider the Yukawa Lagrangian as a perturbation of a type $\mathrm{X}$ model in terms of FCNC couplings. Therefore, we diagonalize the $Y_{2}^{u}, Y_{2}^{d}$, and $Y_{1}^{\ell}$ matrices, where $Y_{1}^{u}, Y_{1}^{d}$, and $Y_{2}^{\ell}$ remain nondiagonal leading to tree-level FCNCs in the Yukawa sector. After diagonalization, the Yukawa Lagrangian involving the neutral scalars can be written as follows:

$$
\begin{aligned}
-\mathcal{L}_{\text {Yukawa }}^{\phi}= & \bar{u}_{L}\left[\left(\frac{c_{\alpha} \mathbf{m}^{u}}{v s_{\beta}}-\frac{c_{\beta-\alpha} \Sigma^{u}}{\sqrt{2} s_{\beta}}\right) h+\left(\frac{s_{\alpha} \mathbf{m}^{u}}{s_{\beta} v}+\frac{s_{\beta-\alpha} \Sigma^{u}}{\sqrt{2} s_{\beta}}\right) H\right] u_{R}+\bar{d}_{L}\left[\left(\frac{c_{\alpha} \mathbf{m}^{d}}{v s_{\beta}}-\frac{c_{\beta-\alpha} \Sigma^{d}}{\sqrt{2} s_{\beta}}\right) h+\left(\frac{s_{\alpha} \mathbf{m}^{d}}{s_{\beta} v}+\frac{s_{\beta-\alpha} \Sigma^{d}}{\sqrt{2} s_{\beta}}\right) H\right] d_{R} \\
& +\bar{e}_{L}\left[\left(-\frac{s_{\alpha} \mathbf{m}^{\ell}}{v c_{\beta}}+\frac{c_{\beta-\alpha} \Sigma^{\ell}}{\sqrt{2} c_{\beta}}\right) h+\left(\frac{c_{\alpha} \mathbf{m}^{\ell}}{c_{\beta} v}-\frac{s_{\beta-\alpha} \Sigma^{\ell}}{\sqrt{2} c_{\beta}}\right) H\right] e_{R} \\
& -i\left[\bar{u}_{L}\left(\frac{\mathbf{m}^{u}}{t_{\beta} v}-\frac{\Sigma^{u}}{\sqrt{2} s_{\beta}}\right) u_{R}+\bar{d}_{L}\left(-\frac{\mathbf{m}^{d}}{t_{\beta} v}+\frac{\Sigma^{d}}{\sqrt{2} s_{\beta}}\right) d_{R}+\bar{e}_{L}\left(\frac{t_{\beta} \mathbf{m}^{\ell}}{v}-\frac{\Sigma^{\ell}}{\sqrt{2} c_{\beta}}\right) e_{R}\right] A+\text { H.c. }
\end{aligned}
$$

Here $\mathbf{m}^{f}=U_{L}^{f} \mathbf{M}^{f} U_{R}^{f}$ are the diagonal mass matrices of the fermions and $U_{L}^{f}$ and $U_{R}^{f}$ are the unitary matrices required to diagonalize $\mathbf{M}^{f}, \quad \Sigma^{u}=U_{L}^{u} Y_{1}^{u} U_{R}^{\dagger u}, \quad \Sigma^{d}=$ $U_{L}^{d} Y_{1}^{d} U_{R}^{\dagger d}$, and $\Sigma^{\ell}=U_{L}^{\ell} Y_{2}^{u} U_{R}^{\dagger l}$. Also, $\quad c_{\alpha}=\cos \alpha$, $s_{\alpha}=\sin \alpha, \quad c_{\beta-\alpha}=\cos (\beta-\alpha), \quad s_{\beta-\alpha}=\sin (\beta-\alpha)$, and $t_{\beta}=\tan \beta$. It can be easily checked that the FCNC processes occur due to the presence of nonzero $\Sigma^{f}$ matrices. When $\Sigma^{f}=0$ the Yukawa couplings in Eq. (9) reduce to the couplings in the type $\mathrm{X}$ 2HDM. Following the convention of Ref. [52], $\Sigma^{f}$ matrices are parametrized in terms of dimensionless free parameters $\chi^{f}$ in the following manner:

$$
\Sigma_{i j}^{f}=\sqrt{m_{i}^{f} m_{j}^{f}} \chi_{i j}^{f} / v
$$

In general, $\chi_{i j}^{f}$ may not be equal to $\chi_{j i}^{f}$, but for simplicity we assume $\chi_{i j}^{f}=\chi_{j i}^{f}$ in our analysis. We mention here that the nondiagonal couplings of the pseudoscalar $A$ [see Eq. (9)] will be crucial for our study and we will call them $y_{\mu e}, y_{\tau e}$, and $y_{\mu \tau}$ in the following sections.

As the rotation matrix for charged scalars is the same as that for pseudoscalars [which can be seen from Eqs. (4) and (5)], the Yukawa couplings of the charged Higgs boson are similar to those of the $C P$-odd scalar and can be written as

$$
\begin{aligned}
\mathcal{L}_{Y}^{H^{ \pm}}= & \frac{\sqrt{2}}{v} \bar{u}_{i}\left(m_{i}^{u}\left(\xi^{u *}\right)_{k i} V_{k j} P_{L}+V_{i k}\left(\xi^{d}\right)_{k j} m_{j}^{d} P_{R}\right) d_{j} H^{+} \\
& +\frac{\sqrt{2}}{v} \bar{\nu}_{i}\left(\xi^{\ell}\right)_{i j} m_{j}^{\ell} P_{R} \ell_{j} H^{+}+\text {H.c. },
\end{aligned}
$$

where the sum over flavor indices is indicated, $V \equiv U_{L}^{u} U_{L}^{d \dagger}$ is the Cabibbo-Kobayashi-Maskawa matrix, and $P_{R, L}=$ $\left(1 \pm \gamma_{5}\right) / 2$ are the chiral projection operators. The expressions for the $\xi^{f}$ matrices are given below:

$$
\xi^{u}=\frac{1}{t_{\beta}} \delta_{i j}-\frac{1}{\sqrt{2} s_{\beta}} \sqrt{\frac{m_{i}^{u}}{m_{j}^{u}}} \chi_{i j}^{u},
$$




$$
\begin{gathered}
\xi^{d}=-\frac{1}{t_{\beta}} \delta_{i j}+\frac{1}{\sqrt{2} s_{\beta}} \sqrt{\frac{m_{i}^{d}}{m_{j}^{d}}} \chi_{i j}^{d}, \\
\xi^{\ell}=t_{\beta} \delta_{i j}-\frac{1}{\sqrt{2} c_{\beta}} \sqrt{\frac{m_{i}^{\ell}}{m_{j}^{\ell}}} \cdot \chi_{i j}^{\ell}
\end{gathered}
$$

Here we can also see that nonzero $\Sigma$ matrices and equivalently nonzero $\xi$ matrices will introduce nontrivial coupling structure even in the charged Higgs interaction with the quarks and leptons. One can check that in the absence of these matrices the couplings reduce to the couplings in the type X $2 \mathrm{HDM}$.

\section{EXPLORATION OF MUON $(g-2)$}

The muon anomalous magnetic moment is one of the biggest triumphs of quantum field theory. A precise measurement of this helps one to comprehend the higher-order corrections contributing to it. Moreover, it indicates the existence of new physics because of the long-standing discrepancy between the SM prediction and experimental observations [3]. The ongoing E989 experiment at Fermilab [4] and future E34 experiment at J-PARC [5] may shed new light on this discrepancy between theory and experiment.

In classical quantum mechanics the value of $g_{\mu}$ (the gyromagnetic ratio for $\mu$ ) is 2 . It receives corrections from loop effects in quantum field theory. These corrections are parametrized in terms of $a_{\mu}=\frac{g_{\mu}-2}{2}$. In the SM it receives contributions via $\mathrm{QED}$, electroweak, and hadronic loops. A great deal of effort has been put into determining the SM prediction to an unprecedented level of accuracy. SM contributions up to three orders in the electromagnetic constant were calculated in Ref. [53-56]. Taking into account pure QED, electroweak, and hadronic contributions, the predicted value for the muon anomaly in the SM is given by [55-75]

$$
a_{\mu}^{\mathrm{SM}}=116591810(43) \times 10^{-11} .
$$

The most recent bound comes from BNL (2006) data [76], which gives

$$
a_{\mu}^{\exp }=116592089(63) \times 10^{-11} .
$$

The difference between theory and experiment denotes a $3.7 \sigma$ discrepancy which can certainly leave room to search for NP scenarios,

$$
\Delta a_{\mu}=a_{\mu}^{\exp }-a_{\mu}^{\mathrm{SM}}=279(76) \times 10^{-11} .
$$

In this work we consider one-loop and two-loop BarZee-type contributions to $\Delta a_{\mu}$ in a generalized 2HDM. It was shown in earlier works $[34,77]$ that the two-loop BarZee diagrams can bring sizable contributions for a large region of parameter space. We present the diagrams for the one-loop contributions to $\Delta a_{\mu}$ in Fig. 1 and the two-loop Bar-Zee diagrams in Figs. 2-4. We mention here that the two-loop Bar-Zee contributions dominate over the oneloop contributions, although the two-loop diagrams have a loop suppression factor as well as an enhancement factor of $\frac{M^{2}}{m_{\mu}^{2}}$, where $M$ is the mass of the heavy particle running in the loop (namely, $t, b, H^{ \pm}$, or $W^{ \pm}$), as can be seen from Fig. 2. This enhancement factor usually dominates over the loop suppression. The Bar-Zee contribution from an internal photon and heavy fermion or $H^{ \pm}$running in the loop has been studied in great detail in the past, and these diagrams give rise to major contributions to $\Delta a_{\mu}$. The contributions from diagrams where a $Z$ boson replaces the internal photon are negligible due to coupling and mass suppression. Also, the diagram involving $W^{ \pm}$in the loop will have a negligible contribution because of the suppression in the coupling between $W^{ \pm}$bosons and the nonstandard $C P$ even Higgs in the alignment limit. We have also considered the Bar-Zee diagrams where a charged Higgs replaces the neutral Higgs and $W^{ \pm}$replaces the internal $\gamma$ in Fig. 3. It has been shown in Ref. [34] that their contributions can be sizable in some regions of the parameter space. In Fig. 4 we also consider the diagrams with an internal charged Higgs or $W^{ \pm}$, where the grey circle represents the same loops as in Fig. 3, excluding the fermion loops for the $W^{ \pm}$diagram, because that will be a pure SM contribution.

We compute $\Delta a_{\mu}$ taking into account all of the aforementioned diagrams, following Refs. [34,77]. Next, we scan the parameter space of our model and plot the allowed

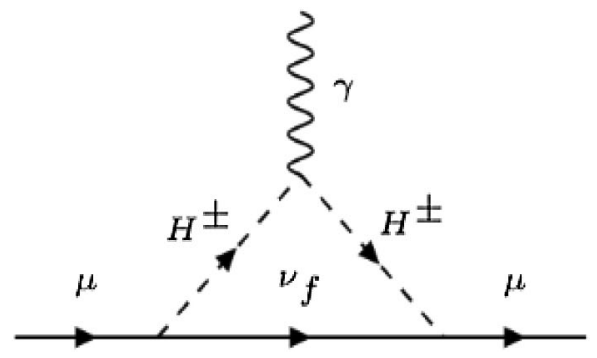

FIG. 1. Nonstandard contribution to $\Delta a_{\mu}$ at one loop. 

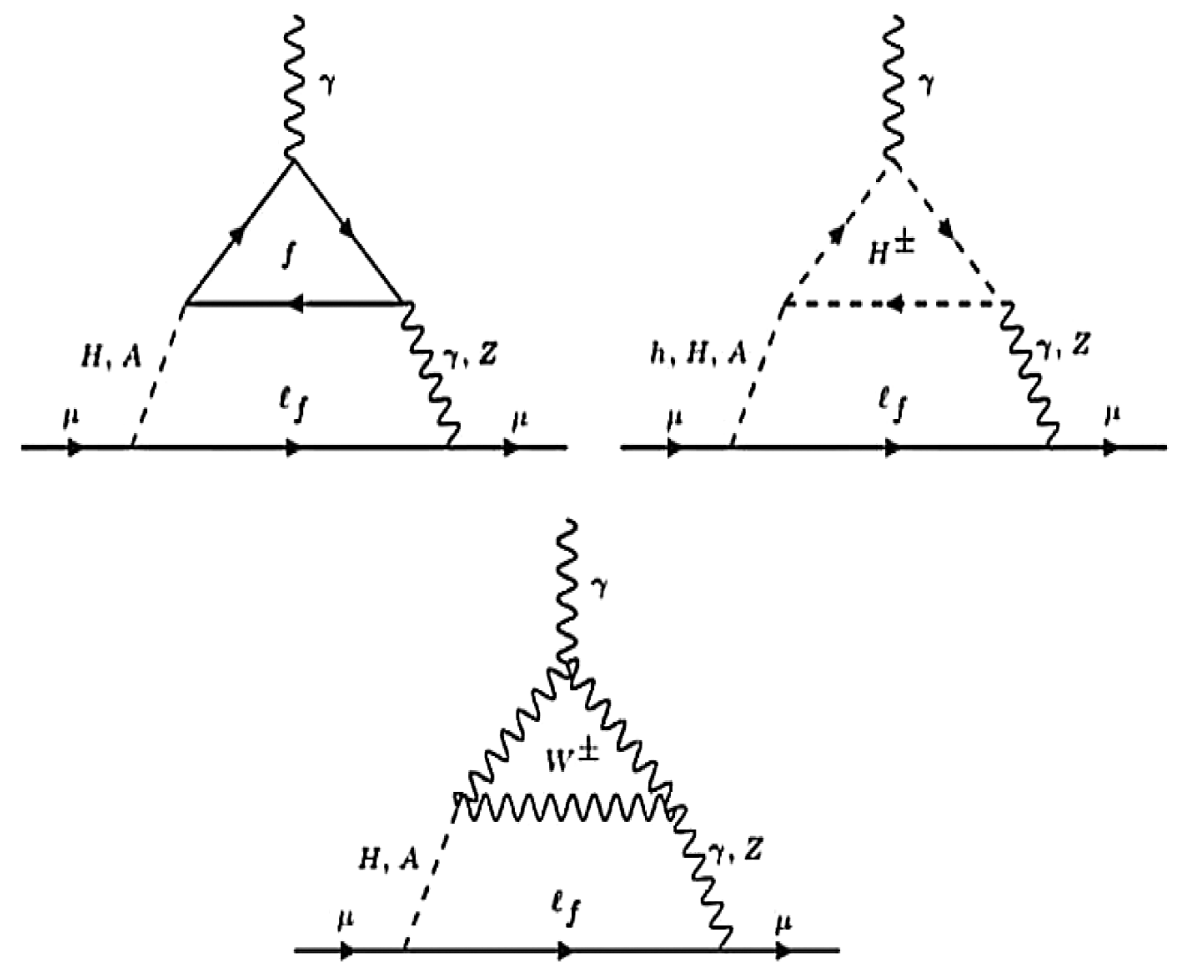

FIG. 2. Nonstandard contribution to $\Delta a_{\mu}$ from two-loop Bar-Zee diagrams with internal $\gamma / Z$.

region in the $m_{A}-\tan \beta$ plane in Fig. 5. For the scan, the flavor-changing couplings are taken to be $y_{\mu e}=10^{-7}$, $y_{\tau e}=5 \times 10^{-5}$, and $y_{\mu \tau}=5 \times 10^{-5}$. The choice of such Yukawa couplings will be discussed in the next section.
The nonstandard neutral $C P$-even Higgs mass and charged Higgs mass are fixed at 120 and $150 \mathrm{GeV}$, respectively. We mention here that the parameter space allowed by $\Delta a_{\mu}$ has been explored in the context of the type X 2HDM [31,39].
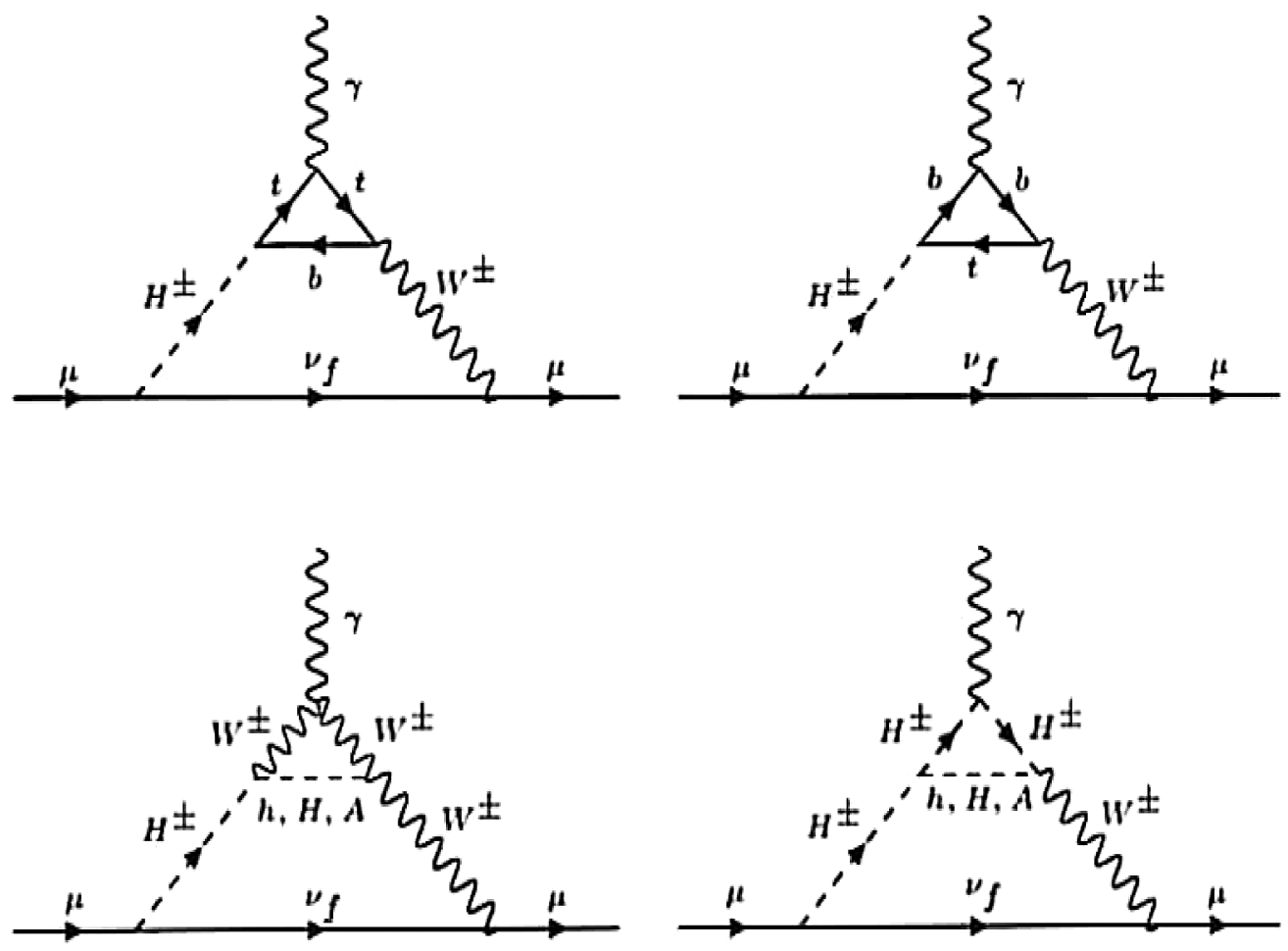

FIG. 3. Nonstandard contribution to $\Delta a_{\mu}$ from two-loop Bar-Zee diagrams with internal $W^{ \pm}$and $H^{ \pm}$. 

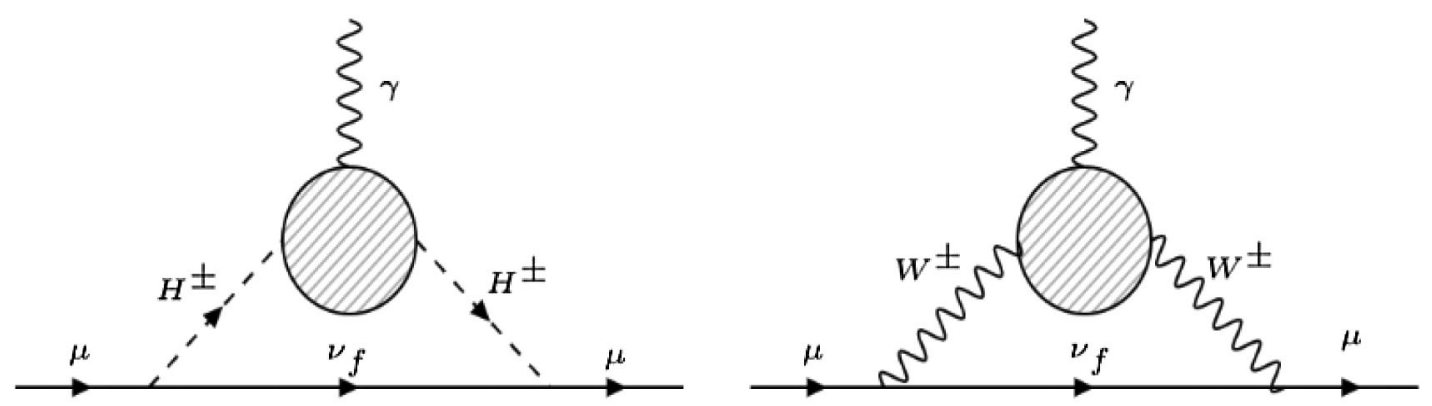

FIG. 4. Nonstandard contribution to $\Delta a_{\mu}$ from two-loop Bar-Zee diagrams as in Fig. 3, but with internal $W^{ \pm}$or $H^{ \pm}$.

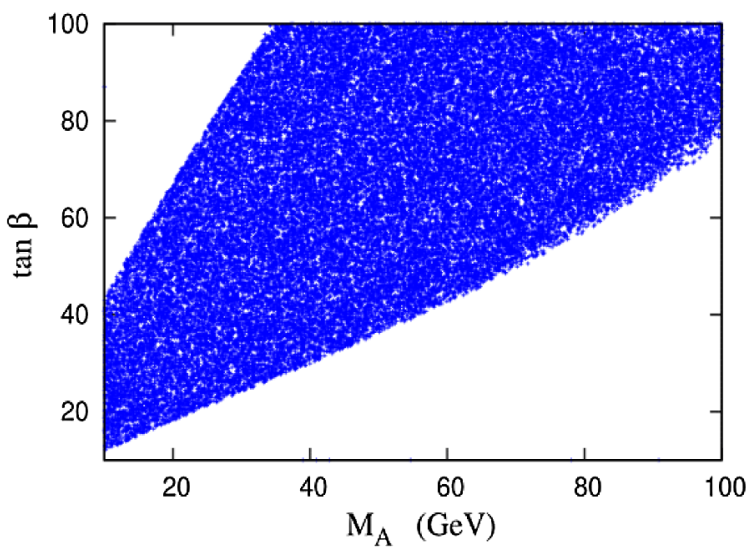

FIG. 5. Allowed region in $m_{A}-\tan \beta$ plane from $g_{\mu}-2$ data at $3 \sigma$. The flavor-changing couplings are taken to be $y_{\mu e}=10^{-7}$, $y_{\tau e}=5 \times 10^{-5}$, and $y_{\mu \tau}=5 \times 10^{-5}$. The nonstandard neutral $C P$-even Higgs mass is $120 \mathrm{GeV}$ and the charged Higgs mass is $150 \mathrm{GeV}$.

We have considered the most updated experimental bound, an exhaustive set of one- and two-loop diagrams, and the effect of lepton-flavor-violating vertices, as compared to the earlier works. One can check that a low-mass pseudoscalar with an enhanced coupling to the $\tau$ leptons will give a significant contribution to $\Delta a_{\mu}$ (see top left panel of Fig. 2). In our model the coupling of the pseudoscalar with a pair of $\tau$ leptons is proportional to $\tan \beta$. Therefore, the low- $m_{A}$ and large-tan $\beta$ region is favored in light of $g_{\mu}-2$ data. While scanning the parameter space we have used the $3 \sigma$ upper and lower bounds on the experimentally observed central value of $\Delta a_{\mu}$ [Eq. (17)].

\section{CONSTRAINTS ON THE MODEL}

We have seen from the discussion in the previous section that the major contribution to the anomalous magnetic moment of the muon comes from the low-mass pseudoscalar contribution at moderate to large $\tan \beta$. However, in the presence of the nondiagonal Yukawa matrices it is inevitable that the similar contributions will also generate FCNC processes. These flavor-changing processes include loop-induced $\mu \rightarrow e \gamma, \tau \rightarrow e \gamma, \tau \rightarrow \mu \gamma, \mu \rightarrow 3 e$, and $\mu-e$ conversions, all of which put a strong constraint on the flavor-changing couplings as well as the (pseudo)scalar masses and $\tan \beta$. It is evident that low-mass pseudoscalar and large-tan $\beta$ regions will be disfavored from the limits coming from the low-energy measurements, which seems to be in tension with the requirement of muon $(g-2)$. We study these constraints carefully in the upcoming subsection and explore the regions of parameter space that are consistent with the limits from the nonobservation of lowenergy flavor-violating processes as well as the experimental observation of $\left(g_{\mu}-2\right)$. Moreover, these flavorchanging vertices also give rice to FCNCs in the (pseudo) scalar-mediated tree-level decays in the leptonic final states. Our main objective in this work is to probe this region of parameter space in collider experiments. We mention here that to get a sufficient event rate in collider experiments we focus on the low mass range of the decaying (pseudo)scalar. We now proceed to discuss various constraints on our model which further guide us to choose our benchmarks for the upcoming direct search analysis in collider experiments.

\section{A. Limits from low-energy measurements}

In the SM, lepton flavor is conserved since neutrinos are massless. In neutrino oscillation [6,7], LFV has been observed in the neutrino sector. However, to date LFV has not been observed in the charged lepton sector. Therefore, lepton flavor violation can be treated as one of the important tools to search for new physics. Many new physics models can accommodate LFV processes. Since no such signal has been observed yet, there are strong limits on these LFV processes [11]. We will soon see that in the low- $m_{A}$ region (which is of our primary interest from the $\left(g_{\mu}-2\right)$ requirements) the LFV processes will also be dominated by the pseudoscalar contribution in the loop. Therefore, these limits from the low-energy LFV processes will essentially constrain the nondiagonal lepton Yukawa couplings of the pseudoscalar A, namely, $y_{\mu e}, y_{\tau e}$, and $y_{\mu \tau}$.

The recent bound on $\operatorname{BR}(\mu \rightarrow e \gamma)<4.2 \times 10^{-13}$ comes from the MEG experiment [12]. The other important constraint on LFV will come from $\mu \rightarrow 3 e$, which is a natural consequence of $\mu \rightarrow e \gamma$ decay when the resulting 

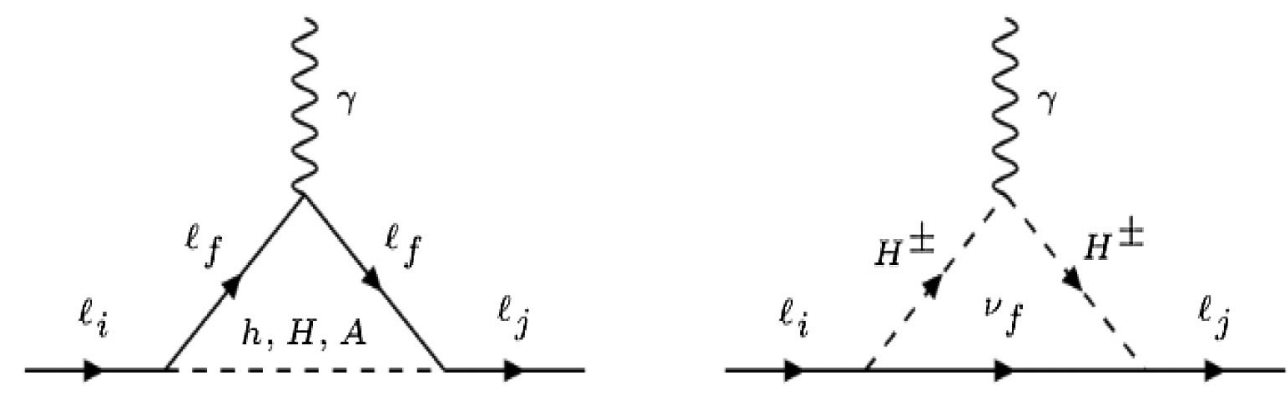

FIG. 6. One-loop contribution to lepton-flavor-violating decays.

photon converts to an $e^{+} e^{-}$pair. Apart from that, $\mu-e$ conversion in nuclei can also be an important signature of LFV. Assuming that the chirality-flipping dipole term dominates, the relation between $\mu \rightarrow e \gamma$ and other possible LFV constraints, namely, $\mu-e$ conversion (CR) and $\operatorname{BR}(\mu \rightarrow 3 e)$, can be roughly estimated as $[10,13]$

$$
\begin{aligned}
\mathrm{CR}(\mu T i \rightarrow e T i) & \simeq \frac{1}{200} \mathrm{BR}(\mu \rightarrow e \gamma), \\
\mathrm{BR}(\mu \rightarrow 3 e) & \simeq \frac{1}{160} \mathrm{BR}(\mu \rightarrow e \gamma) .
\end{aligned}
$$

We emphasize that the relations quoted in Eq. (18) are model dependent [78], and here we merely try to give a rough order-of-magnitude estimation of the observables. If we try to translate the limits according to the relations in Eq. (18), we find that the limit on $\mathrm{CR}(\mu T i \rightarrow e T i)$ has to be $<2.1 \times 10^{-15}$ for it to be of the same strength as the limit from $\mu \rightarrow e \gamma$, while from experiment [14] this upper limit is $6.1 \times 10^{-13}$ [9]. A similar argument holds for $\mathrm{BR}(\mu \rightarrow 3 e)$. From Eq. (18), $\mathrm{BR}(\mu \rightarrow 3 e)$ should be $<2.62 \times 10^{-15}$ for this upper limit to be of the same strength as $\operatorname{BR}(\mu \rightarrow e \gamma)$, while the experimental upper bound is $1.0 \times 10^{-12}$ [8]. Therefore, it is evident that these two constraints are relatively weak. Hence, for our analysis we take into account the strongest bound, which comes from $\mu \rightarrow e \gamma .{ }^{4}$

Similar to the $\mu-e$ sector, there are strong constraints on the $(\tau \rightarrow e \gamma)$ and $(\tau \rightarrow \mu \gamma)$ branching ratios. The current bound on $\operatorname{BR}(\tau \rightarrow e \gamma)<3.3 \times 10^{-8}[11]$ and $\mathrm{BR}(\tau \rightarrow \mu \gamma)<$ $4.4 \times 10^{-8}$ [11] puts a strong constraint on $y_{\tau e}$ and $y_{\mu \tau}$, respectively. One should also take into account the limits on $\operatorname{BR}(\tau \rightarrow 3 e)<2.7 \times 10^{-8} \quad[15]$ and $\operatorname{BR}(\tau \rightarrow 3 \mu)<$ $2.1 \times 10^{-8}[15]$. However, compared to $\operatorname{BR}(\tau \rightarrow e \gamma)$, the limit on $\operatorname{BR}(\tau \rightarrow 3 e)$ is weaker due to an additional suppression of factor $\alpha$. The same is true for the limit on

\footnotetext{
${ }^{4}$ However, just as a passing comment we would like to highlight the fact that the next generation of experiments such as Mu2e and COMET will use aluminum as a target, aiming at a sensitivity of $\sim 10^{-17}$ on $\mu-e$ conversion [79], which would probably be the strongest constraint among all of the LFV observables.
}

$\operatorname{BR}(\tau \rightarrow 3 \mu)$ which imposes a much weaker limit compared to $\operatorname{BR}(\tau \rightarrow \mu \gamma)$.

We calculate the LFV processes at one and two loops following Refs. $[13,45]$. The presence of flavor-nondiagonal Yukawa matrices in the generalized 2HDM gives rise to a flavor-violating coupling between scalars and leptons at the tree level. This in turn enables the LFV decays at one and two loops. We show the corresponding one-loop diagrams in Fig. 6. One can see that these diagrams are the same as those in Fig. 1 with modifications in the incoming and outgoing fermion lines. Likewise, the twoloop diagrams taking part in LFV decays can be obtained from Figs. 2-4 by similar modifications. It is worth mentioning that the loop contribution from the neutral scalars dominates over the contribution from the charged scalar loop (see Fig. 6). We have found that the two-loop contribution to the $\tau \rightarrow \mu \gamma$ and $\tau \rightarrow e \gamma$ amplitudes is a mere $\sim 2 \%$ of its one-loop counterpart. On the contrary, in the case of $\mu \rightarrow e \gamma$, the addition of the two-loop contribution induces a factor of 3 enhancement of the one-loop amplitude.

It is interesting to note that $\mathrm{BR}(\tau \rightarrow e \gamma)$ and $\mathrm{BR}(\tau \rightarrow \mu \gamma)$ constrain the couplings $y_{\tau e}$ and $y_{\mu \tau}$, respectively. The reason behind this is the following. The major contributions to the corresponding amplitudes come from the $\tau$ loop (see left panel of Fig. 6), where $y_{\tau e}$ and $y_{\mu \tau}$ appear with $m_{\tau}$. Therefore, these terms dominate over the other terms which are accompanied by $m_{\mu}$ and $m_{e}$ or are product of two LFV couplings. Hence, the upper limit on the aforementioned branching ratios particularly constrains $y_{\tau e}$ and $y_{\mu \tau}$. However, the situation is different in the case of $\mathrm{BR}(\mu \rightarrow e \gamma)$. In this case, the $\tau$ loop has the highest contribution in terms of the loop integral. The $\tau$-loop integral at one loop comes with a coefficient $y_{\mu \tau} \times y_{\tau e}$. Hence, its contribution can be comparable with the $e$ or $\mu$ loop, with coefficients $y_{\mu e}$ multiplied by $m_{e}$ or $m_{\mu}$. Therefore, $\operatorname{BR}(\mu \rightarrow e \gamma)$ is not solely controlled by $y_{\mu e}$ at one loop. However, at two loops it is the $y_{\mu e}$ coupling that dominates the amplitude.

We have seen that $\operatorname{BR}(\tau \rightarrow e \gamma)$ constrains $y_{\tau e}<10^{-4}$ and $\operatorname{BR}(\tau \rightarrow \mu \gamma)$ constrains $y_{\tau \mu}<10^{-4}$. However, for $y_{\mu e}$ the situation is not so straightforward. Unlike $\tau \rightarrow e \gamma$ and 
$\tau \rightarrow \mu \gamma$, the decay $\mu \rightarrow e \gamma$ does not primarily constrain $y_{\mu e}$ coupling, as discussed earlier. After the inclusion of twoloop in the amplitude calculation, the coupling $y_{\mu e}$ gets a strong upper bound $\left(<10^{-6}\right)$ in order to satisfy all the three LFV constraints.

It is important to note that the branching ratios we just described also depend strongly on the scalar masses and $\tan \beta$, along with the flavor-changing couplings. In Figs. $7-10$ we show the regions allowed by LFV constraints in the $m_{A}-\tan \beta$ plane for specific choices of flavor-changing Yukawa couplings. In Figs. 7-10 we also superimpose the region allowed by $\left(g_{\mu}-2\right)$ data onto the region allowed by low-energy LFV data.

It can be clear from Figs. 7 and 8 that the two limits tend to favor nonoverlapping regions, unless the LFV Yukawa couplings are below certain values. In Figs. 9 and 10 we present our choice of LFV Yukawa couplings, for which we get an overlapping region that is allowed by $\left(g_{\mu}-2\right)$ as well as low-energy LFV constraints. We specifically concentrate on the scenario depicted in Fig. 10, because the values of flavor-violating Yukawa couplings $\left(y_{\mu e}=10^{-7}\right.$, $\left.y_{\tau e}=5 \times 10^{-5}, y_{\mu \tau}=5 \times 10^{-5}\right)$ in this case produce an adequate event rate which can be probed at the HL-LHC. Therefore, this region is of primary interest to us from the collider point of view.

\section{B. Theoretical constraints}

The constraints from the requirements of vacuum stability and perturbativity have been studied in detail in earlier works $[31,80]$. It has been pointed out that a large separation between $m_{A}$ and $m_{H}^{ \pm}$is disfavored from the theoretical considerations of vacuum stability and perturbativity. Since we are interested in the low-mass pseudoscalars from the requirements of $\left(g_{\mu}-2\right)$, it is imperative to check the upper limit on $m_{H}^{ \pm}$that is compatible with low $m_{A}$. The vacuum stability and perturbativity conditions put bounds on the $\lambda$ parameters and thereby correlate the masses of different neutral and charged scalars. The vacuum stability condition requires $[80,81]$
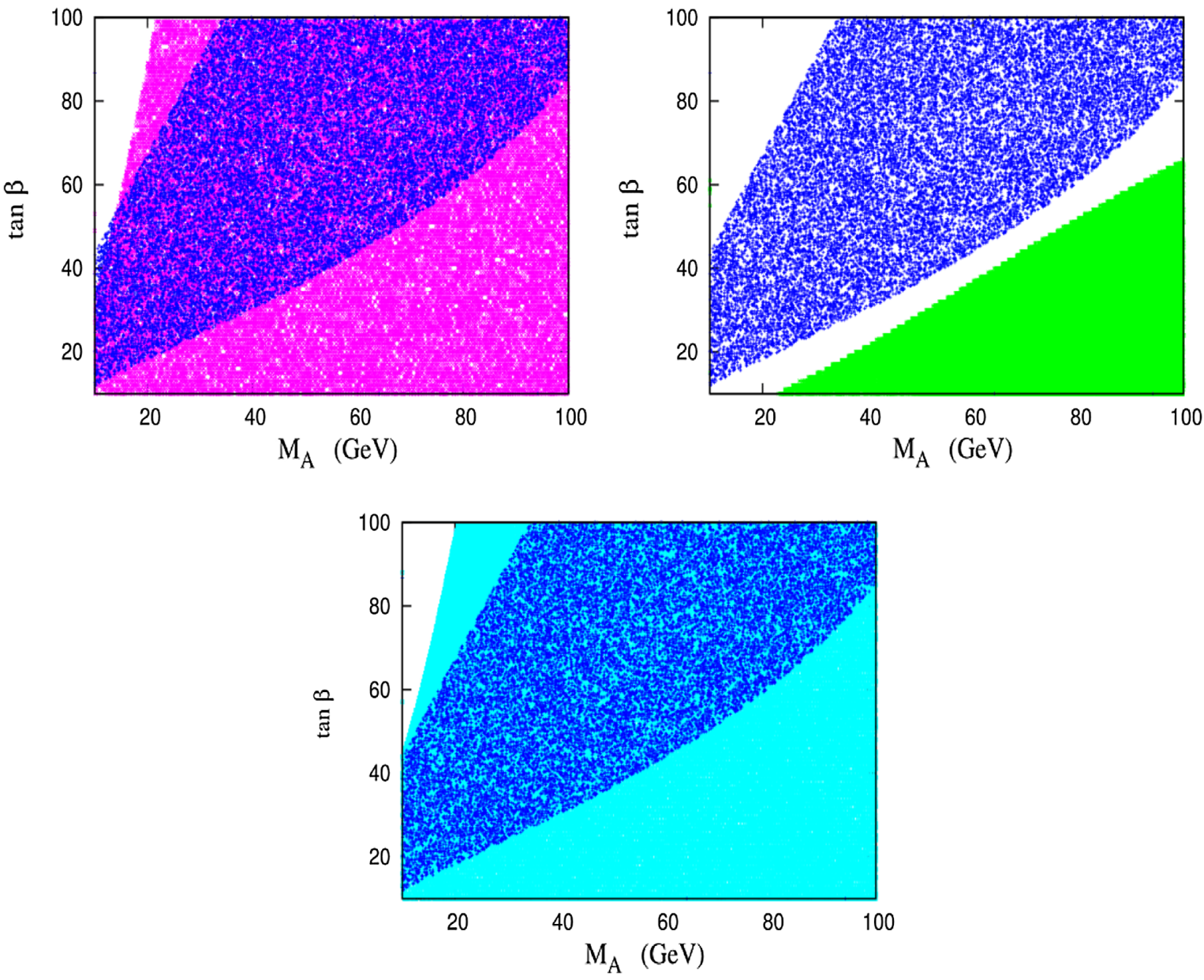

FIG. 7. The magenta, green, and cyan regions are the allowed ranges for $\mu \rightarrow e \gamma, \tau \rightarrow e \gamma$, and $\tau \rightarrow \mu \gamma$, respectively. The blue band is the $3 \sigma$ allowed range for the muon anomaly. The flavor-changing couplings are taken to be $y_{\mu e}=10^{-7}, y_{\tau e}=10^{-4}$, and $y_{\mu \tau}=10^{-5}$. The nonstandard neutral $C P$-even Higgs mass is $120 \mathrm{GeV}$ and the charged Higgs mass is $150 \mathrm{GeV}$. 

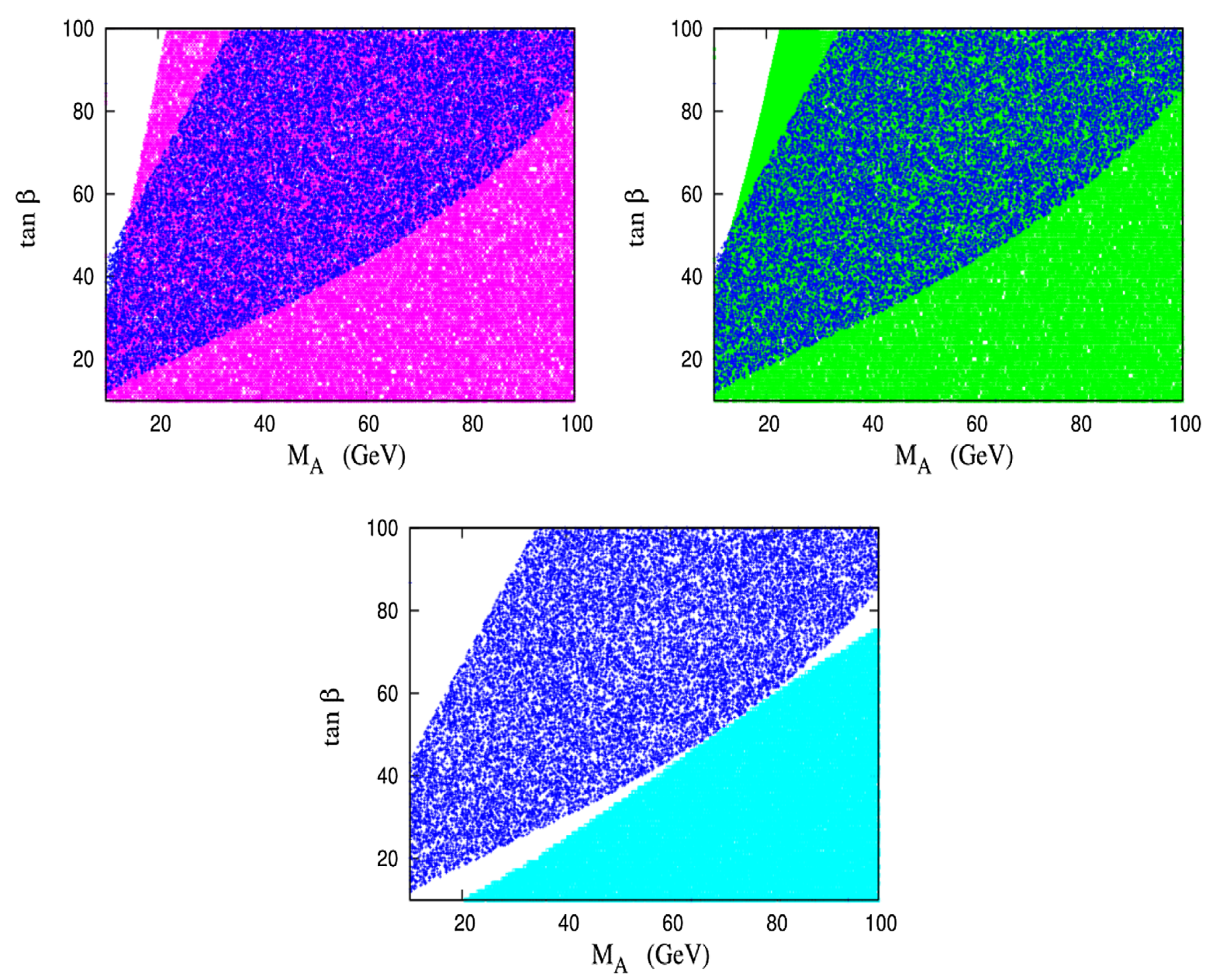

FIG. 8. The magenta, green, and cyan regions are the allowed ranges for $\mu \rightarrow e \gamma, \tau \rightarrow e \gamma$, and $\tau \rightarrow \mu \gamma$, respectively. The blue band is the $3 \sigma$ allowed range for the muon anomaly. The flavor-changing couplings are taken to be $y_{\mu e}=10^{-7}, y_{\tau e}=10^{-5}$, and $y_{\mu \tau}=10^{-4}$. The nonstandard neutral $C P$-even Higgs mass is $120 \mathrm{GeV}$ and the charged Higgs mass is $150 \mathrm{GeV}$.

$$
\begin{gathered}
\lambda_{1}>0, \quad \lambda_{2}>0, \quad \lambda_{3}>-\sqrt{\lambda_{1} \lambda_{2}}, \\
\lambda_{3}+\lambda_{4}-\left|\lambda_{5}\right|>\sqrt{\lambda_{1} \lambda_{2}} .
\end{gathered}
$$

The resulting squared-masses for the $C P$-odd and charged Higgs states are given by [80]

$$
\begin{gathered}
m_{A}^{2}=\frac{m_{12}^{2}}{s_{\beta} c_{\beta}}-\frac{1}{2} v^{2}\left(2 \lambda_{5}+\frac{\lambda_{6}}{t_{\beta}}+\lambda_{7} t_{\beta}\right), \\
m_{H^{ \pm}}^{2}=m_{A}^{2}+\frac{1}{2} v^{2}\left(\lambda_{5}-\lambda_{4}\right) .
\end{gathered}
$$

It is clear from Eq. (21) that the mass-squared difference $m_{H}^{ \pm 2}-m_{A}^{2}$ is proportional to $\lambda_{5}-\lambda_{4}$, which should be less than $\lambda_{3}+\sqrt{\lambda_{1} \lambda_{2}}$. Along with the vacuum stability criteria, the requirement of perturbativity, i.e., that all of the quartic couplings $C_{H_{i} H_{j} H_{k} H_{l}}<4 \pi$, puts an upper limit on $m_{H}^{ \pm}-m_{A}$. The parameter space allowed by stability and perturbativity constraints when $m_{h}=125 \mathrm{GeV}$ was indicated in Ref. [31]. We have performed a scan in the following range of parameters for the scenario where the mass of the heavier $C P$-even neutral Higgs $m_{H}=125 \mathrm{GeV}$ (the justification behind this choice will be discussed shortly) and the hard $Z_{2}$-symmetry-breaking parameters $\lambda_{6}$ and $\lambda_{7}$ are assumed to be nonzero. We have assumed the alignment limit in the analysis and therefore have varied the mixing angle $\cos (\beta-\alpha)$ close to unity:

$m_{A} \in[10.0 \mathrm{GeV}, 60.0 \mathrm{GeV}], m_{H} \in[62.5 \mathrm{GeV}, 125.0 \mathrm{GeV}]$, $m_{H}^{ \pm} \in[89.0 \mathrm{GeV}, 190.0 \mathrm{GeV}], \quad m_{12}^{2} \in\left[-1000 \mathrm{GeV}^{2}\right.$, $\left.1000 \mathrm{GeV}^{2}\right], \quad \tan \beta \in[10,70], \quad|\cos (\beta-\alpha)| \in[0.99,1]$, $\lambda_{6} \in[0,0.1], \lambda_{7} \in[0,0.1]$.

In Fig. 11 we show the parameter space allowed by stability, unitarity, and perturbativity constraints. We show only the low- $m_{A}$ region as we will be interested in this region in our collider analysis. In the left panel of Fig. 11 we show the upper limit of $m_{H^{ \pm}}$as a function of $m_{A}$, as pointed out in the foregoing discussion. We can see that $m_{H^{ \pm}}<170-180 \mathrm{GeV}$ is allowed for low $m_{A}$. In the right panel of Fig. 11 we show the constraints in the $\tan \beta-m_{A}$ 

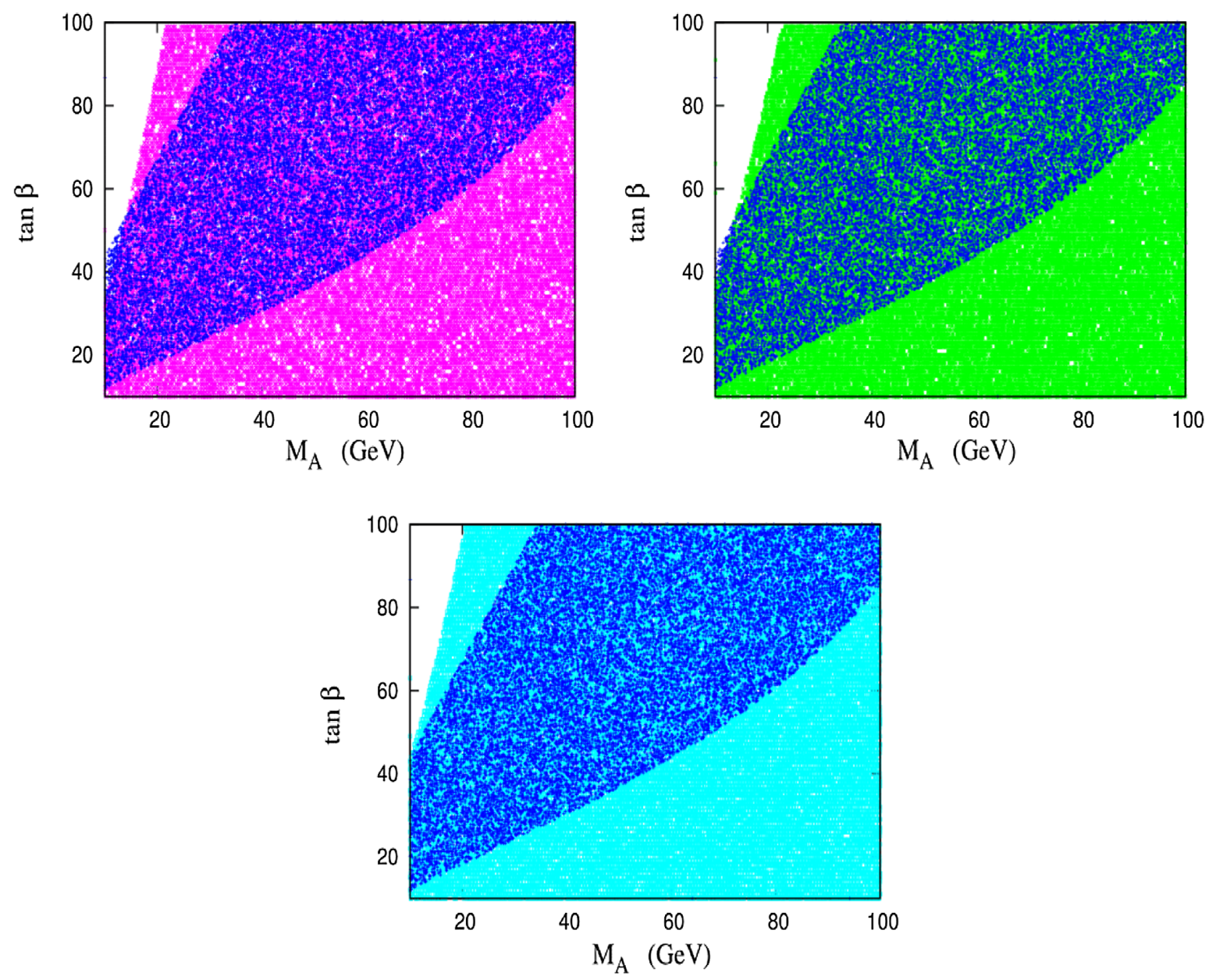

FIG. 9. The magenta, green, and cyan regions are the allowed ranges for $\mu \rightarrow e \gamma, \tau \rightarrow e \gamma$, and $\tau \rightarrow \mu \gamma$, respectively. The blue band is the allowed $3 \sigma$ allowed range for the muon anomaly. The flavor-changing couplings are taken to be $y_{\mu e}=10^{-7}, y_{\tau e}=10^{-5}$, and $y_{\mu \tau}=10^{-5}$. The nonstandard neutral $C P$-even Higgs mass is $120 \mathrm{GeV}$ and the charged Higgs mass is $150 \mathrm{GeV}$.

plane. We see that although a very large $\tan \beta$ is allowed from perturbativity considerations, low to moderate $\tan \beta$ values are much more favored compared to higher values.
Using the relations between the quartic couplings $\lambda$ and the physical masses and Higgs mixing parameter $m_{12}^{2}$, one can find the $h A A$ coupling $[39,80]$

$$
\begin{aligned}
g_{h A A}= & \frac{1}{2 v}\left[\left(2 m_{A}^{2}-m_{h}^{2}\right) \frac{\cos (\alpha-3 \beta)}{\sin 2 \beta}+\left(8 m_{12}^{2}-\sin 2 \beta\left(2 m_{A}^{2}+3 m_{h}^{2}\right)\right) \frac{\cos (\beta+\alpha)}{\sin ^{2} 2 \beta}\right] \\
& +v\left[\sin 2 \beta \cos 2 \beta\left(\lambda_{6}-\lambda_{7}\right) \sin (\beta-\alpha)-\left(\lambda_{6} \sin \beta \sin 3 \beta+\lambda_{7} \cos \beta \cos 3 \beta\right) \cos (\beta-\alpha)\right] .
\end{aligned}
$$

It is important to notice that the low- $m_{A}$ region of parameter space that we are interested in yields a substantial branching fraction for $h \rightarrow A A$ decay, where $h$ is the $125 \mathrm{GeV}$ Higgs and $m_{A}<\frac{m_{h}}{2}$. The experimental upper limit on this branching ratio is rather strong [82], where a stringent limit comes from the search for the $(p p \rightarrow h \rightarrow A A)$ process in the $\mu^{+} \mu^{-} \tau^{+} \tau^{-}$final state. The only way such a small branching ratio can be achieved is when the coupling $g_{h A A}$ is extremely small. This in turn imposes a relation between $m_{12}^{2}, \tan \beta$, and $m_{A}$ [83]. However, $m_{12}^{2}$ is a crucial parameter that ensures perturbativity. It is required for perturbativity that $m_{12}^{2} \sim \frac{m_{H}^{2}}{\tan \beta}$. It was shown in Ref. [83] that in the case where $125 \mathrm{GeV}$ Higgs is the lightest Higgs boson, and $m_{H}>125 \mathrm{GeV}$, it is possible to obey the perturbativity constraints as well as the upper limit on $\operatorname{BR}(h \rightarrow A A)$ for a low $\tan \beta<10$ and a mass gap $m_{H}-m_{h}$ that is not very large. Although this region is phenomenologically viable, the $\left(g_{\mu}-2\right.$ requirements (see Fig. 5) impose that $m_{A}$ should also be very small, i.e., $m_{A}<10 \mathrm{GeV}$. This statement is only valid in the 

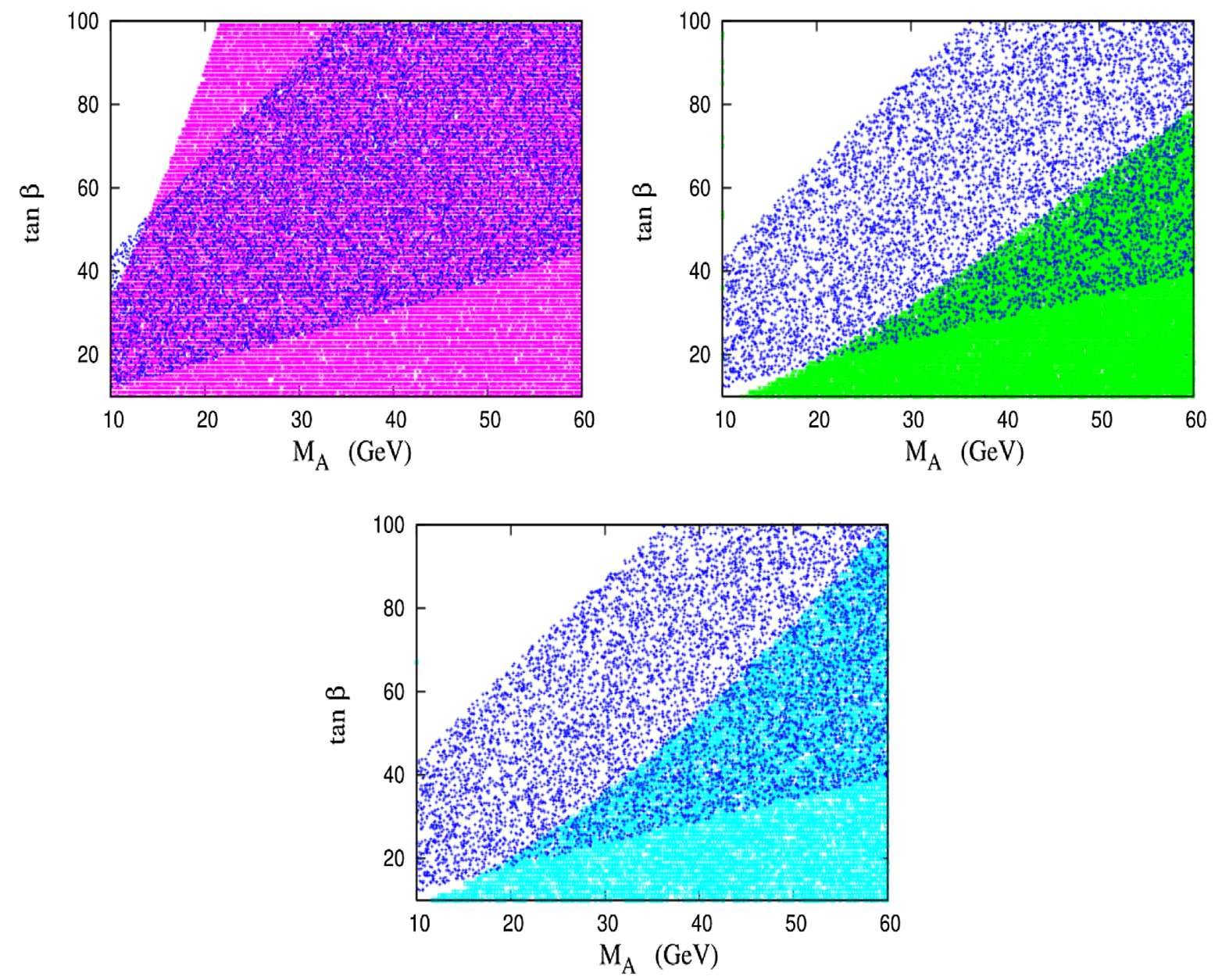

FIG. 10. The magenta, green, and cyan regions are the allowed range for $\mu \rightarrow e \gamma, \tau \rightarrow e \gamma$, and $\tau \rightarrow \mu \gamma$, respectively. The blue band is the allowed $3 \sigma$ allowed range for the muon anomaly. The overlapping regions satisfy both constraints. The flavor-changing couplings are taken to be $y_{\mu e}=10^{-7}, y_{\tau e}=5 \times 10^{-5}$, and $y_{\mu \tau}=5 \times 10^{-5}$. The nonstandard neutral $C P$-even Higgs mass is $120 \mathrm{GeV}$ and the charged Higgs mass is $150 \mathrm{GeV}$.

"right-sign" region of 2HDM where the Higgs couplings with the fermions and gauge bosons are of same sign. The so-called "wrong-sign" region where the Higgs couplings to the fermions and gauge bosons are of opposite sign gives rise to an entirely different allowed region and

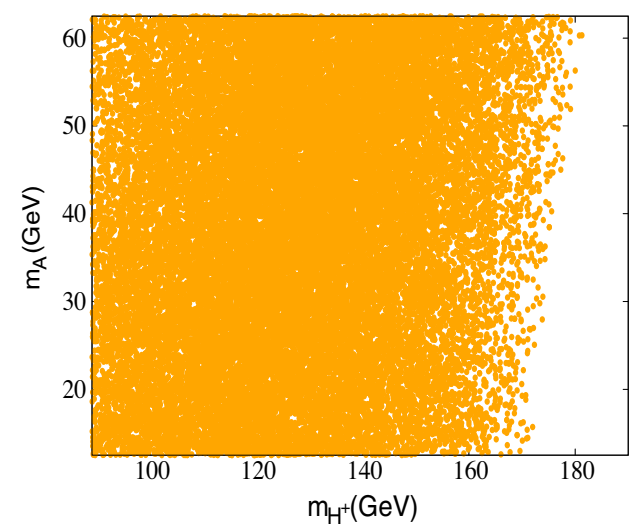

phenomenological signatures. We do not explore this scenario in the current work, and it will be considered in a future study.

The other possibility is to consider the case when the heavier $C P$ even Higgs is SM-like, i.e., $m_{H}=125 \mathrm{GeV}$.

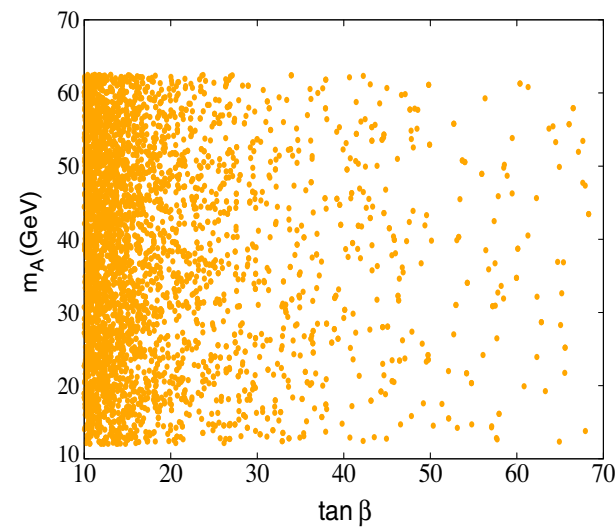

FIG. 11. Parameter space allowed by stability, unitarity, and perturbativity constraints. 
However, in this case the LEP limit implies that either $m_{A}$ or $m_{h}$ can be $<\frac{m_{H}}{2}$. We consider the low-mass pseudoscalar, and therefore $m_{h}>\frac{m_{H}}{2}$. Here, as in the previous case, the limit on $\operatorname{BR}(h \rightarrow A A)$ will indicate an extremely small value of the coupling $g_{H A A}$ whose expression is given as follows:

$$
\begin{aligned}
g_{H A A}= & \frac{1}{2 v}\left[\left(2 m_{A}^{2}-m_{H}^{2}\right) \frac{\cos (\alpha-3 \beta)}{\sin 2 \beta}+\left(8 m_{12}^{2}-\sin 2 \beta\left(2 m_{A}^{2}+3 m_{H}^{2}\right)\right) \frac{\cos (\beta+\alpha)}{\sin ^{2} 2 \beta}\right] \\
& +v\left[\sin 2 \beta \cos 2 \beta\left(\lambda_{6}-\lambda_{7}\right) \cos (\beta-\alpha)+\left(\lambda_{6} \sin \beta \sin 3 \beta+\lambda_{7} \cos \beta \cos 3 \beta\right) \sin (\beta-\alpha)\right] .
\end{aligned}
$$

In this case, there is more freedom compared to the previous case in terms of the allowed parameter space. One can have a pseudoscalar mass $>10 \mathrm{GeV}$ with a moderate $\tan \beta$, with suitable values of $m_{12}^{2}$ and $m_{h}$, while simultaneously satisfying the perturbativity condition and obtaining a small $\mathrm{BR}(H \rightarrow A A)$. From this point onward, we will explore this particular scenario, i.e., for our work $m_{H}=125 \mathrm{GeV}$.

\section{Electroweak constraints}

In this section we analyze the impact of constraints arising from electroweak precision measurements on our model, especially the oblique parameters $[84,85]$. The Gfitter Group [86] has published a contour in the plane of $S$ and $T$ parameter taking into account the correlation between them. The status of the 2HDM in light of the most recent global electroweak data has been presented in Ref. [87]. We mention here that we have used the elliptic contour which has been computed with $U$ as a free parameter. This choice leaves us with a less constrained parameter space compared to the cases with $U=0$.

We have calculated the oblique parameters in our model and obtained the allowed region of parameter space at $3 \sigma$. Here we also concentrate on the low- $m_{A}$ region and consider the case $m_{H}=125 \mathrm{GeV}$, i.e., the second lightest $C P$-even Higgs is SM-like. $m_{H^{ \pm}}$has been varied from

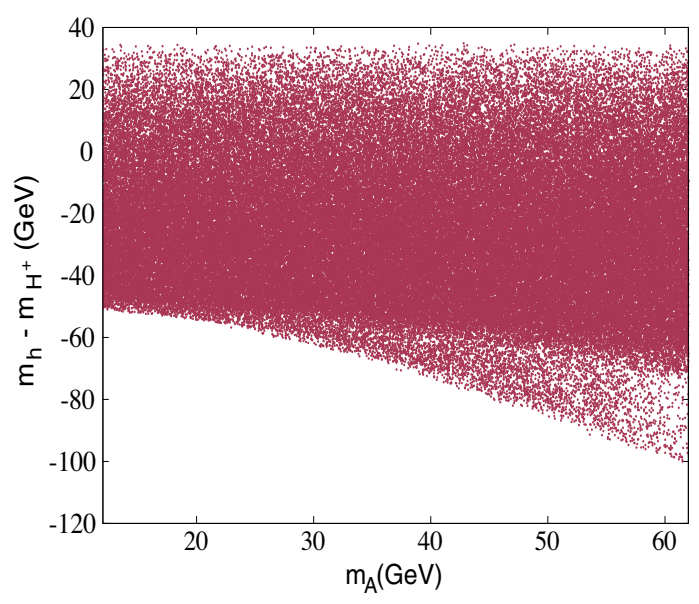

FIG. 12. Parameter space satisfying electroweak constraints in the plane of $m_{A}$ and $m_{h}-m_{H^{ \pm}}$.
90-200 GeV. In Fig. 12 we show the allowed region in the plane of $m_{A}$ and $\left(m_{h}-m_{H^{ \pm}}\right)$. It is evident from the figure that as the pseudoscalar mass decreases, the mass difference $m_{h}-m_{H^{ \pm}}$should also decrease to obey the constraints from oblique parameters. We would like to point out that we have chosen the scalar masses $m_{h}=120 \mathrm{GeV}$ and $m_{H^{ \pm}}=150 \mathrm{GeV}$ (where the neutral $C P$-even heavier Higgs mass $m_{H}$ is $125 \mathrm{GeV}$ ) as representative points in the earlier part of our analysis and also for collider studies. These choices are governed by the requirement of the simultaneous satisfaction of both theoretical and electroweak constraints.

\section{Constraints from $B$ physics}

From the discussions in Sec. II, it is clear that in the presence of flavor-changing couplings in the Yukawa sector the charged Higgs couplings to quarks and leptons are also modified. With new free parameters in the Lagrangian, interesting phenomenologies are likely to show up in rare decay processes that were suppressed in the SM. One such possibility is the rare processes involving $B$ mesons. The free parameters of the model are constrained by the experimental upper bounds on such rare FCNC processes. It is clear from Eq. (10) that the FCNCs within the first two generations are naturally suppressed by the small quark masses, while a larger freedom is allowed for the FCNCs in the top- and bottom-quark sectors. In our analysis, we have also taken only $\lambda_{t t}$ and $\lambda_{b b}$ to be nonzero, where $\lambda_{t t}$ and $\lambda_{b b}$ are the $h t \bar{t}$ and $h b \bar{b}$ coupling strengths, respectively, considering $h$ to be the non-SM-like $C P$-even Higgs.

The rare processes involving $B$ mesons primarily include the decays $B \rightarrow X_{s} \gamma, B_{s} \rightarrow \mu^{+} \mu^{-}$, and $B^{ \pm} \rightarrow \tau^{ \pm} \nu_{\tau}$, and $B^{0}-\bar{B}^{0}$ mixing whose strength is determined by the mass difference $\Delta M_{B}$ between the two states. The most stringent constraint comes from the decay $B \rightarrow X_{s} \gamma$. The impact of these constraints in terms of specific types of $2 \mathrm{HDM}$ as well as a generalized 2HDM have been studied in great detail in earlier works [88-90]. In conventional type I and type II $2 \mathrm{HDMs}$, the dominant additional contribution to the loop-induced decay $B \rightarrow X_{s} \gamma$ comes from the charged Higgs boson-top quark penguin diagrams and its contribution depends on $m_{H^{ \pm}}$. In type II $2 \mathrm{HDMs}$, this extra contribution interferes constructively with its SM counterpart and therefore the lower bound on the charged Higgs 
boson becomes rather high $\left(m_{H}^{ \pm} \gtrsim 600 \mathrm{GeV}\right)$. In type I, the charged Higgs penguin diagram's contribution interferes destructively with its SM counterpart and gives a negligible result at large $\tan \beta$. Therefore, no strong constraint appears on the mass of the charged Higgs in type I models. The type X model has the same structure as type I, as far as the interactions of Higgs with the quark sector are concerned. Therefore, type $\mathrm{X}$ models also do not receive any strong lower bound on $m_{H^{ \pm}}$. As we can think of our model as a perturbation from the type $\mathrm{X}$ scenario, in the absence of the extra terms in the Yukawa Lagrangian there is no strict lower bound on the charged Higgs mass. However, even in the presence of nonzero FCNC Yukawa matrix elements, it is possible to have a low enough charged Higgs mass [21,39,44,47,91] with suitable choices of $\lambda_{t t}$ and $\lambda_{b b}$ couplings. In our analysis we use $\lambda_{t t} \sim 0.5$ and $\lambda_{b b} \sim 2$, which allows a charged Higgs mass $m_{H^{ \pm}} \gtrsim 150 \mathrm{GeV}$.

Another decay process that can constrain our model parameter space is $B^{ \pm} \rightarrow \tau^{ \pm} \nu_{\tau}$, where the charged Higgs enters at the tree level itself. The observed branching ratio for the process $B_{u}^{ \pm} \rightarrow \tau^{ \pm} \nu_{\tau}=(1.06 \pm 0.19) \times 10^{-4}$ [92]. Although it has not yet been observed, the decay $B_{c}^{ \pm} \rightarrow$ $\tau^{ \pm} \nu_{\tau}$ puts an upper limit $(<30 \%)$ [92] on the branching ratio for this decay. However, we have assumed that only $\lambda_{t t}$ and $\lambda_{b b}$ are nonzero in the quark sector, and we find that these limits essentially reduce to a limit on $\lambda_{b b}$ and $\tan \beta$. In Ref. [47] it was shown that $\lambda_{b b} \sim 2$ is favored for large or moderate $\tan \beta$.

The constraint from $\Delta M_{B}$ puts an an upper limit on $\lambda_{t t}$ as a function of the charged Higgs mass [21]. $m_{H^{ \pm}} \gtrsim$ $150 \mathrm{GeV}$ is allowed for $\lambda_{t t} \lesssim 0.5$. Therefore, our choice of parameter space obeys this constraint as well.

The upper limit on $\operatorname{BR}\left(B_{s} \rightarrow \mu^{+} \mu^{-}\right)$is $2.4_{-0.7}^{+0.9} \times 10^{-9}$ [93]. This particular branching fraction constrains the low$\tan \beta(<2)$ region for low $m_{H}^{ \pm}(\sim 100 \mathrm{GeV})$ [89]. For higher charged Higgs masses this limit is further relaxed.

\section{E. Constraints from direct searches at colliders}

Constraints can be obtained from collider searches for the production and decay of on-shell neutral and charged Higgs bosons. There have been numerous searches in the past in this direction. The LEP experiments have looked for pair production of charged Higgs bosons in the process $e^{+} e^{-} \rightarrow \gamma / Z \rightarrow H^{+} H^{-}$. In this process all of the couplings that appear are essentially gauge couplings. Therefore, the predictions for this process depend only on the charged Higgs mass $m_{H^{ \pm}}$. However, the decay and branching fractions of $H^{ \pm}$are indeed model dependent, but a combined search for $H^{ \pm}$in the $\tau \nu$ and $c \bar{s}$ channels put a robust lower limit of $80 \mathrm{GeV}$ on $m_{H^{ \pm}}$[94]. This limit only mildly depends on $\operatorname{BR}\left(H^{ \pm} \rightarrow \tau \nu\right)$.

At the LHC the charged Higgs searches can be categorized into two types. For $m_{H}^{ \pm}<m_{t}$, a charged Higgs can be produced from the decay of a top quark $\left(t \rightarrow b H^{ \pm}\right)$. This decay has been searched for in $\tau \nu[95,96]$ and $c \bar{s}$ [97,98] final states. These searches have put an upper limit on $\mathrm{BR}\left(t \rightarrow b H^{ \pm}\right) \times\left(H^{ \pm} \rightarrow \tau \nu / c \bar{s}\right)$. The other important search mode at the LHC is $\left(p p \rightarrow t b H^{ \pm}\right)$in the final states $\tau \nu$ [96,99], $c \bar{s}$ [100,101], and $t \bar{b}$ [102].

Collider searches for the nonstandard neutral Higgs also put constraints on the parameter space of interest. Searches for nonstandard Higgs bosons are performed at the LHC in various channels with SM final states. As we are specifically interested in the low-pseudoscalar-mass region with enhanced coupling to leptons, the limits that are crucial for our case come from the search for low mass pseudoscalar produced in association with $b$ quarks and decaying into a $\tau \tau$ final state $[103,104]$. Constraints from the search for lowmass (pseudo)scalars produced in association with $b \bar{b}$ and decaying into $b \bar{b}[105,106]$ has been taken into account. CMS has also searched for decays involving two nonstandard Higgs bosons, such as $h / H \rightarrow Z(\ell \ell) A(\tau \tau)$ [107] and $h / H \rightarrow Z(\ell \ell) A(b \bar{b})$ [108]. However, these limits become applicable for heavier $C P$-even Higgs $\gtrsim 200 \mathrm{GeV}$. Therefore, these particular searches do not have any considerable affect on our parameter space.

We mention here that one should also take into account the limits coming from the direct search for the $125 \mathrm{GeV}$ Higgs in various final states, including $\tau \tau$ [109,110], and $\mu \mu$ $[111,112]$. Moreover, as the focus of our work is FCNCs in the Yukawa sector, the constraints coming from flavorviolating decays of the $125 \mathrm{GeV}$ Higgs boson also put constraints on the flavor-violating Yukawa matrix elements. The CMS experiments have looked for the $125 \mathrm{GeV}$ Higgs decaying to $e \mu$ and $e \tau$ final states [113]. CMS also puts an upper limit on the branching ratio for the $125 \mathrm{GeV}$ Higgs decaying to a $\mu \tau$ final state [114]. Undoubtedly, these limits are crucial for our study. However, as we strictly confine ourselves to the alignment limit $[\cos (\beta-\alpha) \approx 0.999]$, the flavor-violating decays of the $125 \mathrm{GeV}$ Higgs $(H$ in our case) will receive a suppression by a factor of $\sin ^{2}(\beta-\alpha)$, which can be seen from Eq. (9). Therefore, in this limit the constraints coming from lepton-flavor-violating decays of the $125 \mathrm{GeV}$ Higgs are trivially satisfied.

An important constraint comes from the direct search for $125 \mathrm{GeV}$ Higgs decaying into two light pseudoscalar final states when it is kinematically allowed. The upper bound on this branching ratio translates into a severe constraint on the parameter space of this model. We have discussed this in detail in Sec. IV B and have taken it into account in our analysis.

\section{COLLIDER SEARCHES}

From our discussions in the previous sections it is clear that the existence of flavor violation in the lepton Yukawa sector gives rise to flavor-violating decays of $\mu$ and $\tau$ leptons. The presence of off-diagonal elements in the 
TABLE I. Benchmark points allowed by all constraints and the corresponding production cross sections of our signal at LO at $14 \mathrm{TeV}$ LHC.

\begin{tabular}{cccccccccc}
\hline \hline & $\tan \beta$ & $m_{A}$ (in $\left.\mathrm{GeV}\right)$ & $m_{h}($ in $\mathrm{GeV})$ & $m_{H}^{ \pm}($in $\mathrm{GeV})$ & $m_{12}^{2}\left(\right.$ in $\left.\mathrm{GeV}^{2}\right)$ & $\lambda_{6}$ & $\lambda_{7}$ & $|\cos (\beta-\alpha)|$ & $\sigma_{\text {prod }}(\sqrt{s}=14 \mathrm{TeV})$ \\
\hline BP1 & 15 & 21 & 120 & 150 & 970 & 0.001 & 0.001 & 0.999 & 0.085 \\
BP2 & 20 & 25 & 120 & 150 & 843 & 0.1 & 0.005 & 0.999 & 0.067 \\
$\mathrm{BP} 3$ & 22 & 27 & 120 & 150 & 775 & 0.01 & 0.0045 & 0.999 & 0.052 \\
\hline \hline
\end{tabular}

Yukawa matrices are the source of the lepton flavor violation in generalized 2HDMs. The flavor-violating decays of leptons are introduced at loop level via flavorviolating couplings between the scalar and leptons at tree level. These flavor-nondiagonal tree-level Yukawa couplings between the scalar and leptons will also give rise to interesting phenomenology at colliders [17-20].

In this work we consider probing the $C P$-odd scalar $A$ in flavor-violating leptonic decay mode in a generalized 2HDM at the HL-LHC. Our signal process is given as

$$
p p \rightarrow A \rightarrow \ell \tau_{\ell^{\prime}}
$$

where $\ell, \ell^{\prime}=e, \mu$ and $\tau_{\ell^{\prime}}$ denotes the leptonic decay of $\tau$. The signal of our interest is $\ell^{+} \ell^{\prime-}+\mathbb{E}_{T}$.

The SM processes that can give rise to similar final states are $\tau \tau / e e / \mu \mu, t \bar{t}, W^{ \pm}+$jets, a diboson, and the SM Higgs $[17,115]$. Out of these backgrounds, the major background in our signal region is $\tau \tau$. Due to its large cross section, $t \bar{t}$ also serves as an important background. In reality, the $t \bar{t}$ leptonic final state turns out to be an irreducible background, whereas the $t \bar{t}$ semileptonic and $W+$ jets background - despite having a significant cross section-is reduced to a large extent by our preselection criteria, which will be discussed shortly. From now on, we indicate the $t \bar{t}$ leptonic channel as the $t \bar{t}$ background unless specified otherwise. The $e e / \mu \mu$ background also has a considerable cross section. However, in our signal region this background contributes only $<5 \%$ of the $\tau \tau$ background. Therefore, we do not discuss this background explicitly. The diboson and SM Higgs background has a much smaller cross section compared to the aforementioned backgrounds and they turn out to be less severe.

For our analysis, we choose three benchmark points that are valid for all of the experimental and theoretical constraints and quote their production cross sections in Table I. We mention here that since the branching ratios of the pseudoscalars decaying to flavor-violating final states is very small $\left[\mathrm{BR}(A \rightarrow \mu \tau) \approx \operatorname{BR}(A \rightarrow \tau e) \approx 10^{-7}\right]$, owing to the smallness of lepton flavor-violating Yukawa couplings, we are compelled to choose a low-mass pseudoscalar that will have considerable production cross section and therefore will be a viable candidate in collider searches.

We first present the cut-based analysis for this channel in the following subsection. Then, we explore the possible improvement of our results with multivariate techniques using an ANN.

\section{A. Cut-based analysis}

The signal events are generated in MadGraph5@NLO [116] and the model file is implemented in FEYNRULES [117]. We generate both signal and SM background events at leading order (LO) in MadGraph5@NLO [116] using the NNPDF3.0 parton distributions [118]. The parton showering and hadronization are done using the built-in PYTHIA [119] within MadGraph. The showered events are then passed through DELPHES(v3) [120] for the detector simulation, where the jets are constructed using the anti- $K_{T}$ jet algorithm with a minimum jet formation radius $\Delta R=0.5$. The isolated leptons are considered to be separated from the jets and other leptons by $\Delta R_{\ell i} \gtrsim 0.4, i=j, \ell$.

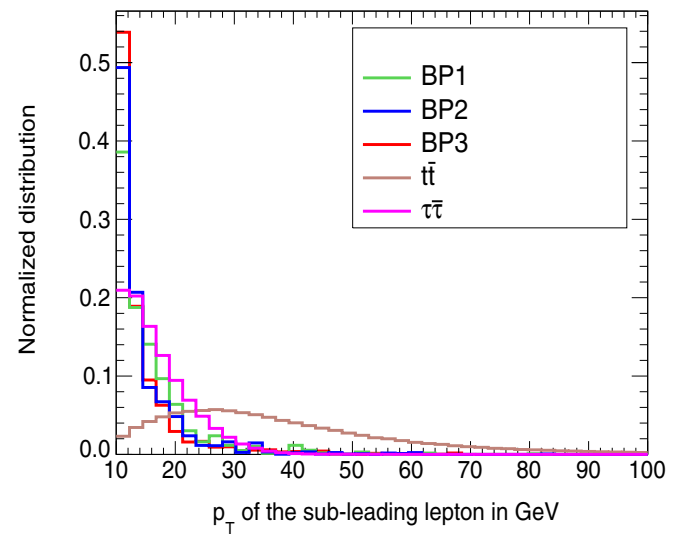

FIG. 13. Distribution of transverse momenta of leading (left) and subleading (right) leptons for signal and backgrounds. 
TABLE II. The cut flow for signal and background and significance reach for our signal at the $14 \mathrm{TeV}^{\mathrm{LHC}}$ for $3 \mathrm{ab}^{-1}$ luminosity.

\begin{tabular}{|c|c|c|c|c|c|c|}
\hline \multirow[b]{2}{*}{ SM-background } & \multicolumn{6}{|c|}{ Effective NLO cross section after the cut (fb) } \\
\hline & Preselection cuts & $\Delta \phi_{\ell \ell^{\prime}}<2.2$ & $M_{\ell \ell^{\prime}}<15 \mathrm{GeV}$ & $\mathscr{E}_{T}<15 \mathrm{GeV}$ & $M_{\text {collinear }}>10 \mathrm{GeV}$ & $M_{T}<25 \mathrm{GeV}$ \\
\hline$\tau \tau$ & 8582.75 & 132.089 & 0.21 & 0.089 & 0.052 & 0.052 \\
\hline$t \bar{t}$ leptonic & 22.10 & 11.01 & 0.099 & 0.016 & 0.016 & 0.0016 \\
\hline \multicolumn{7}{|l|}{ Signal } \\
\hline $\mathrm{BP} 1$ & 0.0689 & 0.0686 & 0.0276 & 0.0266 & 0.0262 & 0.0258 \\
\hline BP2 & 0.0637 & 0.0542 & 0.0081 & 0.0076 & 0.0073 & 0.0073 \\
\hline \multirow[t]{5}{*}{ BP3 } & 0.0513 & 0.0381 & 0.0028 & 0.0026 & 0.0025 & 0.0025 \\
\hline & \multicolumn{2}{|c|}{ Benchmark points } & \multicolumn{4}{|c|}{ Significance reach at $3 \mathrm{ab}^{-1}$ luminosity } \\
\hline & \multicolumn{2}{|l|}{ BP1 } & \multicolumn{4}{|c|}{$5.7 \sigma$} \\
\hline & \multicolumn{2}{|l|}{ BP2 } & \multicolumn{4}{|c|}{$1.7 \sigma$} \\
\hline & \multicolumn{2}{|l|}{ BP3 } & \multicolumn{3}{|c|}{$0.6 \sigma$} & \\
\hline
\end{tabular}

To generate our signal and background events, we employ the following preselection cuts:

$$
\begin{aligned}
& p_{T}(j, b)>20 \mathrm{GeV} ; \quad|\eta(j)|<4.7 ; \quad|\eta(b)|<2.5, \\
& p_{T}(\ell)>10 \mathrm{GeV}, \quad|\eta(\ell)|<2.5 \text {. }
\end{aligned}
$$

The $b$ jets are tagged with the $p_{T}$-dependent $b$-tag efficiency following the criteria of Ref. [121], which has an average $75 \%$ tagging efficiency of the $b$ jets with $50 \mathrm{GeV}<p_{T}<200 \mathrm{GeV}$ and $1 \%$ mistagging efficiency for light jets.

Additionally, we propose the following selection cuts on certain kinematic observables to disentangle the signal from the SM backgrounds that would enhance the signal significance. We describe these observables below.

(1) $p_{T}$ of the leptons: In Fig. 13 we show the transverse momentum $p_{T}$ of the leading and subleading leptons. For the signal, the leptons coming from the decay of a low-mass pseudoscalar tend to have low $p_{T}$. Since the distributions are mostly overlapping for both signal and $\tau \tau$ background, it is very difficult to apply any hard $p_{T}$ cut. However, to affirm that our signal has two isolated leptons, we reject any third lepton with $p_{T}(\ell)>10 \mathrm{GeV}$. Moreover, since our signal is hadronically quiet, we apply a jet veto of $p_{T}(j)>20 \mathrm{GeV}$. We also reject any $b$ jet with $p_{T}(b)>20 \mathrm{GeV}$. This is our preselection cut, as described in Table II. This helps us reduce the $t \bar{t}$ semileptonic and $W^{ \pm}+$jets background.

(2) Selecting $\mathscr{E}_{T}$ : For the signal, neutrino is coming from the leptonic decay of $\tau$. The $\tau$ comes from the decay of the low-mass pseudoscalar. Therefore, for the signal the $\mathbb{E}_{T}$ tends to be small. For the $\tau \tau$ background, the neutrinos are produced almost back to back. So, the lower $\mathbb{E}_{T}$ bins are populated both for the signal and $\tau \tau$ background. On the other hand, as top decay is a three-body decay, the $\mathbb{E}_{T}$ produced in $t \bar{t}$ events peaks at a larger value. We present the distribution of $\mathscr{E}_{T}$ in the left panel of Fig. 14.

(3) Invariant mass of the dilepton pair: In the right panel of Fig. 14 we show the invariant mass of the dilepton pair $M_{\ell \ell^{\prime}}$. In the case of the signal, the leptons come from the decay of the low-mass pseudoscalar, and

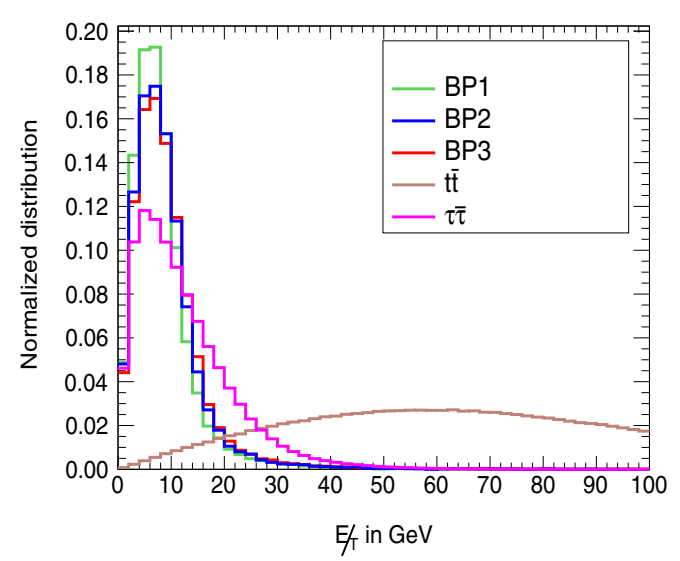

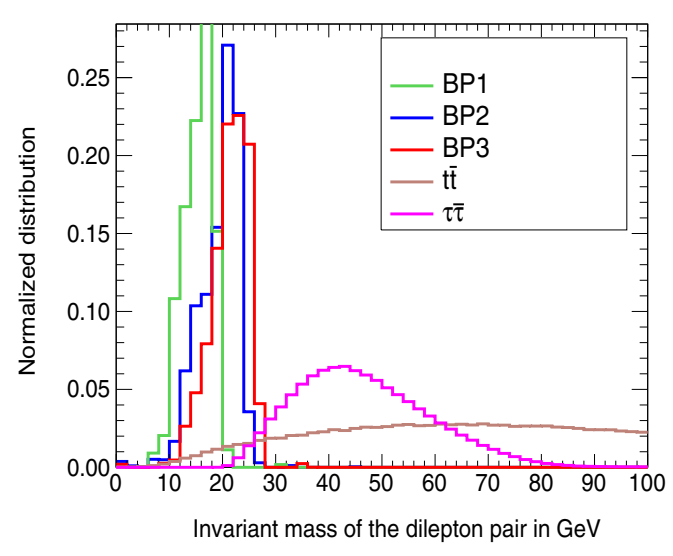

FIG. 14. Distribution of $\mathbb{E}_{T}$ (left) and invariant mass of two leptons for signal and backgrounds. 

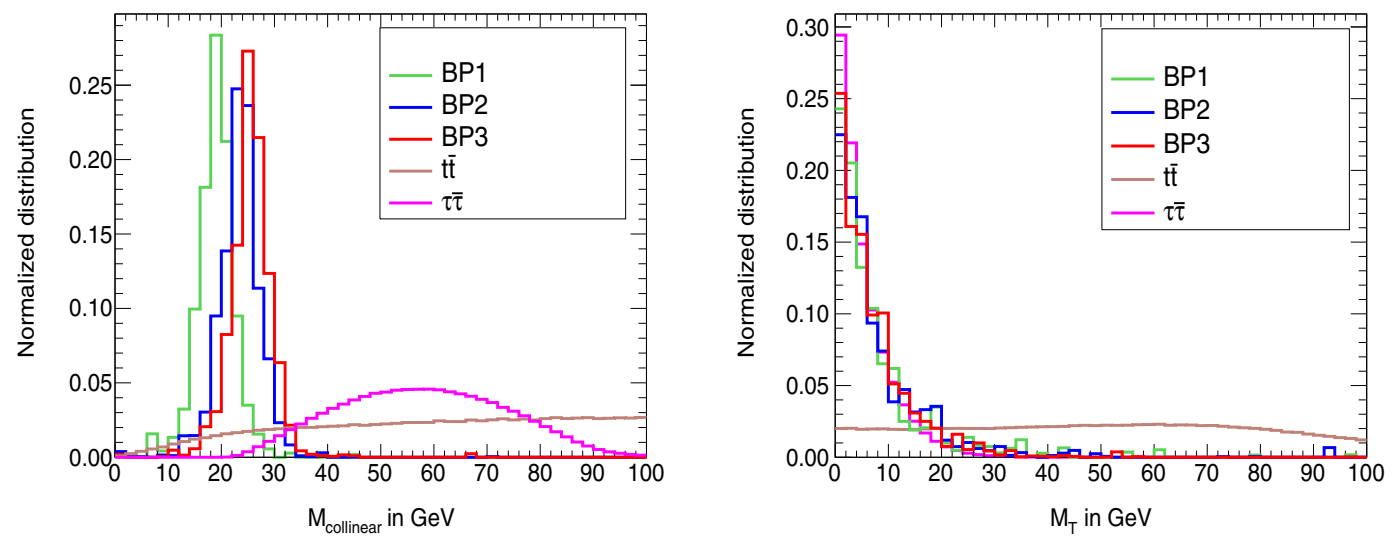

FIG. 15. Distribution of collinear mass (left) and transverse mass (right) for signal and backgrounds.

therefore its distribution peaks at a lower value compared to the $\tau \tau$ and $t \bar{t}$ backgrounds. We mention here that this observable plays a crucial role in reducing the $e e / \mu \mu$ background to a large extent. The invariant mass for $e e / \mu \mu$ peaks at the $Z$-boson mass, and therefore a suitable cut on this variable helps us to get rid of this background. In addition, $M_{\ell \ell^{\prime}}$ turns out to be an important observable to discriminate between $\tau \tau$ background and signal.

(4) Collinear mass: The collinear mass is defined as follows:

$$
m_{A}=M_{\text {collinear }}=\frac{M_{\text {vis }}}{\sqrt{x_{\tau_{\text {vis }}}}}
$$

with the visible momentum fraction of the $\tau$ decay products being $x_{\tau_{\text {vis }}}=\frac{\left|\vec{p}_{T}^{\tau_{\text {vis }}}\right|}{\left|\vec{p}_{T}^{\text {vis }}\right|+\left|\vec{p}_{T}^{\nu}\right|}$, where $\vec{p}_{T}^{\nu}=\left|\vec{E}_{T}\right| \hat{p}_{T}^{\tau_{\text {vis }}}$ and $M_{\mathrm{vis}}$ is the visible mass of the $\tau-\ell$ system. The variable $M_{\text {collinear }}$ essentially reconstructs the mass of the pseudoscalar. From the left panel of Fig. 15, it is clear that $M_{\text {collinear }}$ yields a clear distinction between the signal and the irreducible $\tau \tau$ background. A suitable cut should be imposed on this variable to reduce the $\tau \tau$ background.

(5) Transverse mass variable: The transverse mass is defined as

$$
M_{T}(\ell)=\sqrt{2 p_{T}(\ell) \vec{E}_{T}\left(1-\cos \Delta \phi_{\vec{\ell}-\overrightarrow{\not ̆}_{T}}\right)}
$$

where $\Delta \phi_{\vec{e}-\vec{\not}_{T}}$ denotes the azimuthal angle between the leading lepton and $\mathscr{E}_{T}$. From the right panel of Fig. 15 it is clear that a cut on the $M_{T}$ variable helps us to reduce the $t \bar{t}$ background.

(6) Angle between the leptons: The angle between two leptons is strictly correlated to the invariant mass. Since for the signal the invariant mass of the dilepton pair is small, the azimuthal angle between the two leptons $\Delta \phi_{\ell \ell^{\prime}}$ appears at a lower value compared to the $\tau \tau$ background where the leptons are produced back to back, and as a result $\Delta \phi_{\ell \ell^{\prime}}$ peaks around $\pi$. It is clear from Fig. 16 that a suitable cut on this observable will enhance the signal-background separation.

With optimized cuts on the aforementioned variables (listed in Table II), we get the signal significance as presented in Table II. The significance [122] is calculated using the formula $\mathcal{S}=\sqrt{2\left[(S+B) \ln \left(1+\frac{S}{B}\right)-S\right]}$, where $S$ and $B$ denote the number of signal and background events after applying all of the cuts. We mention here that we multiply the signal and background cross sections by respective $k$ factors to take into account the next-toleading-order (NLO) effects. For the signal we use a $k$ factor of 2 [123], while for $t \bar{t}$ and $\tau \tau$ backgrounds we use a $k$ factor of 1.6 [124] and 1.15 [125], respectively.

\section{B. Improved analysis with an artificial neural network}

Having performed the cut-based analysis, we proceed to analyze the same channel (dilepton $+\mathbb{E}_{T}$ ) with an ANN [126] at the LHC. We explore the possibility of improving

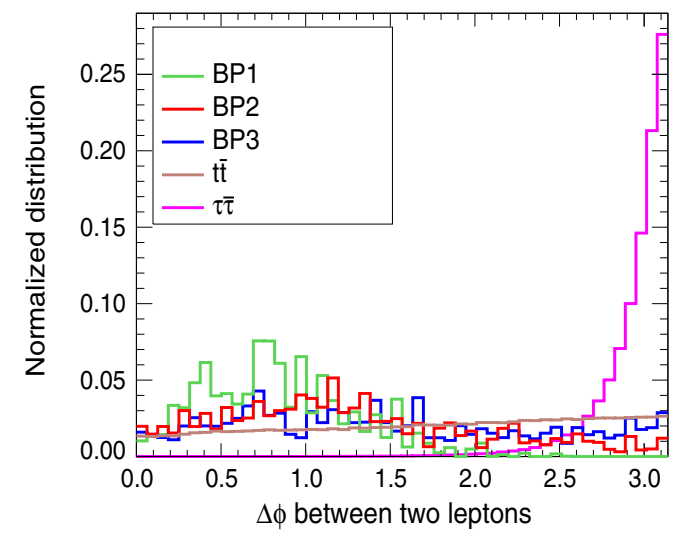

FIG. 16. Distribution of $\Delta \phi$ between two leptons for signal and backgrounds. 
TABLE III. Feature variables for training in the ANN analysis.

\begin{tabular}{lc}
\hline \hline Variable & Definition \\
\hline$p_{T}^{\ell_{1}}$ & Transverse momentum of the leading lepton \\
$p_{T}^{\ell_{1}}$ & Transverse momentum of the subleading lepton \\
$E_{T}^{\text {miss }}$ & Missing transverse energy \\
$M_{\ell \ell^{\prime}}$ & Invariant mass of the dilepton pair \\
$\Delta \phi_{\ell \ell^{\prime}}$ & Azimuthal angle difference between the dilepton pair \\
$\Delta R_{\ell \ell^{\prime}}$ & $\Delta R$ separation between the dilepton pair \\
$M_{\text {vis }}$ & Visible mass of the dilepton system \\
$x_{\text {vis }}$ & Visible momentum fraction of the $\tau$ decay products \\
$M_{\text {collinear }}$ & Collinear mass \\
$M_{T}$ & Transverse mass \\
$\Delta \phi_{\ell_{1} \phi_{T}}$ & Azimuthal angle difference between the \\
$\Delta \phi_{\ell_{2} \not \phi_{T}}$ & Azimuthal angle difference between the subleading \\
\hline \hline
\end{tabular}

our results. This method has been used extensively in the recent past and it has been able to improve the results of cut-based analyses, enabling better separation between the signal and backgrounds. Significant work has been done in the context of the Higgs sector [127-131]. In collider searches for dark matter this methods has also been proven to be extremely useful $[130,132]$. Therefore, we employ this tool in our present analysis where the signal yield is small because of the minuscule branching fraction $\left(\sim 10^{-7}\right)$ of the flavor-changing decay of the pseudoscalar and the distinction between signal and background becomes crucial. We have examined and computed the maximum signal significance for the benchmarks that we considered, which are achievable at the HL-LHC using these technique. The toolkit used for the ANN analysis is the PYTHON-based deep-learning library Keras [133].

From our analysis in the previous section we identify the important input variable that provides a substantial distinction between signal and backgrounds. We mention here that the choice of input variables plays a crucial role. In Table III we present all of the input variables that we have used for our analysis.

For the ANN analysis we have chosen a network with four hidden layers with activation curve ReLU (Rectied Linear Units) at all of them. The batch size is taken to be 1000 and the number of epochs is 100 in our case for each batch. We have used $80 \%$ of the data set for training and $20 \%$ for validation. One possible demerit of these techniques is overtraining of the data sample. In the case of overtraining the training sample indeed gives extremely good accuracy but the validation sample fails to achieve the same degree of accuracy as that of the training sample. However, we have explicitly checked that with our choice of network parameters the algorithm does not overtrain.

We find that the variables $M_{\ell \ell^{\prime}}, M_{\text {collinear }}, M_{T}, \Delta \phi_{\ell \ell^{\prime}}$, and $\Delta R_{\ell \ell^{\prime}}$ play crucial roles in signal-background

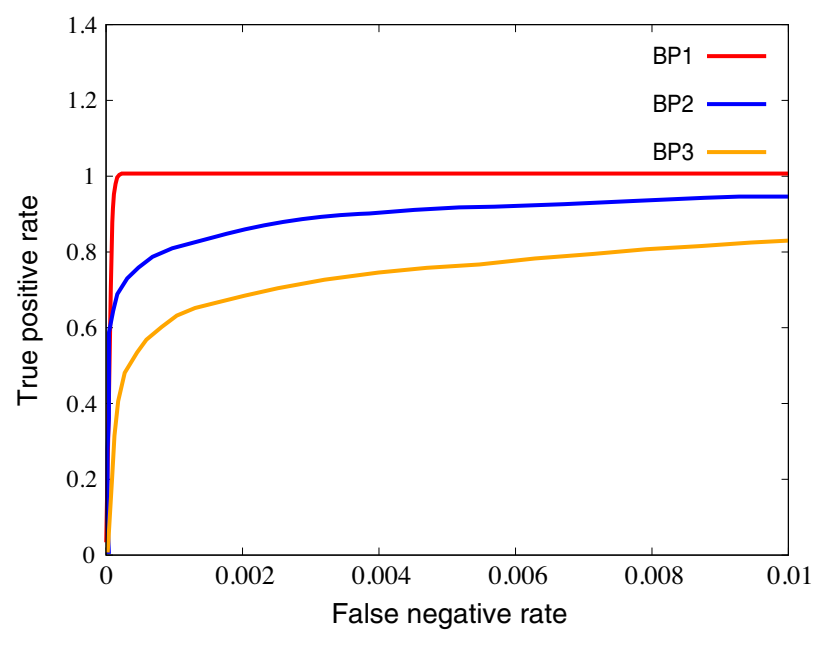

FIG. 17. ROC curves for BP1, BP2, and BP3.

separation. However, there is a strong correlation between $\Delta R_{\ell \ell^{\prime}}, \Delta \phi_{\ell \ell^{\prime}}$, and $M_{\ell \ell^{\prime}}$, as we have already discussed in the previous subsection. We mention here that in order to obtain better performance from the network we have applied two basic cuts, namely, $M_{\ell \ell^{\prime}}<30 \mathrm{GeV}$ and $M_{\text {collinear }}<40 \mathrm{GeV}$ on signal and background events over and above the lepton selection and jet veto. From our discussion of the cut-based analysis we know that these cuts guide us towards the so-called signal region. Therefore, the network will be better trained to separate signal from background specifically in the signal region, and this results in better accuracy in the output. The accuracies we get are 99\% (BP1), 98\% (BP2), and 96\% (BP3) which indicates very good discriminating power between signal and background. In Fig. 17 we show the receiver operating characteristic (ROC) curve for our three benchmark points.

The area under each curve is 0.999 (BP1), 0.998 (BP2), and $0.994(\mathrm{BP} 3)$. We plot only the part of the ROC curves that are relevant for our analysis. We scan over the ROC curve and choose suitable points which yield the maximum signal significance. In Table IV we present the signal significance $\mathcal{S}$ for all of the signal benchmarks we have considered.

Comparing the results of the ANN in Table IV and the cut-based results in Table II, we can see that our analysis with the ANN significantly improves upon the results of the cut-based analysis.

TABLE IV. Signal significance for the benchmark points at $14 \mathrm{TeV}$ with $\mathcal{L}=3 \mathrm{ab}^{-1}$ with cuts + ANN.

\begin{tabular}{lc}
\hline \hline BP & $\mathcal{S}($ cuts + ANN $)$ \\
\hline BP1 & $9.2 \sigma$ \\
BP2 & $5.3 \sigma$ \\
BP3 & $3.2 \sigma$ \\
\hline \hline
\end{tabular}




\section{CONCLUSION}

The motivation behind this work is a much-desired reconciliation between the observed muon anomaly and LFV constraints. In this regard, we considered an extension of the SM with an extended scalar sector, namely, a generalized 2HDM. The additional nonstandard scalars of this model take part in the muon anomaly, and flavornondiagonal Yukawa matrices lead to LFV processes. We have shown that the long-standing problem of the muon anomaly and LFV constraints can be solved simultaneously over a considerable range of parameter space in this model. We show such a region in Fig. 10 with flavor-changing couplings fixed at $y_{\mu e}=10^{-7}, y_{\tau e}=5 \times 10^{-5}$, and $y_{\mu \tau}=$ $5 \times 10^{-5}$ and the nonstandard $C P$-even and charged Higgs masses are fixed at 120 and $150 \mathrm{GeV}$, respectively, where both the muon anomaly and LFV constraints are satisfied.

We then implemented theoretical constraints pertaining to the requirements of perturbativity, unitarity, and vacuum stability. The flavor-nondiagonal Yukawa matrices are also severely constrained by the $B$-physics observables. The direct searches for the SM Higgs as well as the additional scalar states in colliders put another set of bounds on the model parameter space. One such crucial direct search constraint turns out to be the search for the SM Higgs decaying to two light pseudoscalars. Our aim in this work was to search for lepton flavor violation in the scalar sector in colliders. Therefore, the scalar states with low mass prove to be the best candidates for such searches owing to their large production cross section. We have also found that it is the light $C P$-odd scalar $(A)$ of our model that helps us to explain the $\left(g_{\mu}-2\right)$ data. The lightness of the pseudoscalar also implies a large branching ratio of the $125 \mathrm{GeV}$ Higgs into a pair of pseudoscalars when the decay is kinematically feasible. To ensure the upper bound to this branching fraction coming from collider data, along with the perturbativity requirements, one is drawn to the scenario where the observed $125 \mathrm{GeV}$ Higgs is the heavier of the two $\mathrm{CP}$-even states of the 2HDM in the alignment limit. However, this statement is valid only in the "rightsign" region of the $2 \mathrm{HDM}$ that we have considered in this work. The "wrong-sign" scenario will give rise to a different allowed region and interesting phenomenology, which we want to pursue in detail in the future.

After determining the region allowed by all constraints, we looked for flavor-violating decays of the $C P$-odd scalar (A) in the $\ell \tau \rightarrow \ell^{+} \ell^{\prime-}+\mathbb{E}_{T}$ final state, where $\tau$ decays leptonically and $\ell, \ell^{\prime}=e, \mu$. Performing a rectangular cutbased analysis for the $14 \mathrm{TeV}$ LHC with $3 \mathrm{ab}^{-1}$ luminosity, we showed that the significance drops from $\sim 6 \sigma$ to $\sim 1 \sigma$ as the mass of the scalar increases from 21 to $27 \mathrm{GeV}$. We then performed an analysis using an ANN and observed significant improvements in our results from the cut-based analysis. We would like to point out that although we have probed a narrow region of parameter space in terms of pseudoscalar mass, we did this in order to investigate the reach of LFV searches at the $14 \mathrm{TeV}$ LHC with $3 \mathrm{ab}^{-1}$ at $\gtrsim 3 \sigma$ significance. The results of the ANN analysis in Table IV indicate that even higher masses of $A$ can be probed, although with somewhat lower significance $(<3 \sigma)$. Also, a further upgrade in the luminosity as well as energy frontier will enable us to probe heavier $C P$-odd scalars decaying into lepton-flavor-violating final states.

\section{ACKNOWLEDGMENTS}

This work was supported by funding available from the Department of Atomic Energy, Government of India, for the Regional Centre for Accelerator-based Particle Physics (RECAPP), Harish-Chandra Research Institute.
[1] G. Aad et al. (ATLAS Collaboration), Observation of a new particle in the search for the Standard Model Higgs boson with the ATLAS detector at the LHC, Phys. Lett. B 716, 1 (2012).

[2] S. Chatrchyan et al. (CMS Collaboration), Observation of a new boson at a mass of $125 \mathrm{GeV}$ with the CMS Experiment at the LHC, Phys. Lett. B 716, 30 (2012).

[3] T. Blum, A. Denig, I. Logashenko, E. de Rafael, B. L. Roberts, T. Teubner et al., The muon $(g-2)$ theory value: Present and future, arXiv:1311.2198.

[4] J. Grange et al. (Muon g-2 Collaboration), Muon $(g-2)$ Technical Design Report, arXiv:1501.06858.

[5] H. Iinuma (J-PARC muon g-2/EDM Collaboration), New approach to the muon $g-2$ and EDM experiment at J-PARC, J. Phys. Conf. Ser. 295, 012032 (2011).
[6] Y. Fukuda et al. (Super-Kamiokande Collaboration), Evidence for Oscillation of Atmospheric Neutrinos, Phys. Rev. Lett. 81, 1562 (1998).

[7] Q. R. Ahmad et al. (SNO Collaboration), Direct Evidence for Neutrino Flavor Transformation from Neutral Current Interactions in the Sudbury Neutrino Observatory, Phys. Rev. Lett. 89, 011301 (2002).

[8] U. Bellgardt et al., 3E with Sindrum-I (Abstract Only).

[9] P. Wintz, Results of the SINDRUM-II experiment, Conf. Proc. C980420, 534 (1998).

[10] Y. Kuno and Y. Okada, Muon decay and physics beyond the standard model, Rev. Mod. Phys. 73, 151 (2001).

[11] B. Aubert et al. (BABAR Collaboration), Searches for Lepton Flavor Violation in the Decays $\tau^{ \pm} \rightarrow e^{ \pm} \gamma$ and $\tau^{ \pm} \rightarrow \mu^{ \pm} \gamma$, Phys. Rev. Lett. 104, 021802 (2010). 
[12] A. M. Baldini et al. (MEG Collaboration), Search for the lepton flavour violating decay $\mu^{+} \rightarrow \mathrm{e}^{+} \gamma$ with the full dataset of the MEG experiment, Eur. Phys. J. C 76, 434 (2016).

[13] M. Lindner, M. Platscher, and F. S. Queiroz, A call for new physics: The muon anomalous magnetic moment and lepton flavor violation, Phys. Rep. 731, 1 (2018).

[14] A. Bartolotta and M. J. Ramsey-Musolf, Coherent $\mu-e$ conversion at next-to-leading order, Phys. Rev. C 98, 015208 (2018).

[15] J. Beringer et al. (Particle Data Group Collaboration), Review of particle physics (RPP), Phys. Rev. D 86, 010001 (2012).

[16] S.-P. Li and X.-Q. Li, Probing new physics signals with symmetry-restored Yukawa textures, Eur. Phys. J. C 80, 268 (2020).

[17] S. Banerjee, B. Bhattacherjee, M. Mitra, and M. Spannowsky, The Lepton Flavour Violating Higgs Decays at the HL-LHC and the ILC, J. High Energy Phys. 07 (2016) 059.

[18] R. Primulando and P. Uttayarat, Probing lepton flavor violation at the $13 \mathrm{TeV}$ LHC, J. High Energy Phys. 05 (2017) 055.

[19] R. Primulando, J. Julio, and P. Uttayarat, Collider constraints on lepton flavor violation in the 2HDM, Phys. Rev. D 101, 055021 (2020).

[20] S. Jana, V. P. K., and S. Saad, Resolving electron and muon $g-2$ within the 2HDM, Phys. Rev. D 101, 115037 (2020).

[21] F. Mahmoudi and O. Stal, Flavor constraints on the twoHiggs-doublet model with general Yukawa couplings, Phys. Rev. D 81, 035016 (2010).

[22] J. L. Diaz-Cruz, A. Diaz-Furlong, and J. H. Montes de Oca, The general two-Higgs doublet extensions of the SM: A saucerful of secrets, arXiv:1010.0950.

[23] Y. Bai, V. Barger, L. L. Everett, and G. Shaughnessy, General two Higgs doublet model (2HDM-G) and large hadron collider data, Phys. Rev. D 87, 115013 (2013).

[24] A. Arhrib, C.-W. Chiang, D. K. Ghosh, and R. Santos, Two Higgs doublet model in light of the Standard Model $H \rightarrow \tau^{+} \tau^{-}$search at the LHC, Phys. Rev. D 85, 115003 (2012).

[25] X. Liu, L. Bian, X.-Q. Li, and J. Shu, Type-III two Higgs doublet model plus a pseudoscalar confronted with $h \rightarrow \mu \tau$, muon $g-2$ and dark matter, Nucl. Phys. B909, 507 (2016).

[26] N. Chakrabarty, C.-W. Chiang, T. Ohata, and K. Tsumura, Charged scalars confronting neutrino mass and muon $g-2$ anomaly, J. High Energy Phys. 12 (2018) 104.

[27] S. Iguro, Y. Omura, and M. Takeuchi, Testing the 2HDM explanation of the muon $g-2$ anomaly at the LHC, J. High Energy Phys. 11 (2019) 130.

[28] E. J. Chun, J. Kim, and T. Mondal, Electron EDM and Muon anomalous magnetic moment in two-Higgs-doublet models, J. High Energy Phys. 12 (2019) 068.

[29] M. Frank and I. Saha, Muon anomalous magnetic moment in two Higgs doublet models with vector-like leptons, Phys. Rev. D 102, 115034 (2020).

[30] J. E. Chun and T. Mondal, Explaining $g-2$ anomalies in two Higgs doublet model with vector-like leptons, J. High Energy Phys. 11 (2020) 077.
[31] A. Broggio, E. J. Chun, M. Passera, K. M. Patel, and S. K. Vempati, Limiting two-Higgs-doublet models, J. High Energy Phys. 11 (2014) 058.

[32] J. Cao, P. Wan, L. Wu, and J. M. Yang, Lepton-specific two-Higgs doublet model: Experimental constraints and implication on Higgs phenomenology, Phys. Rev. D 80, 071701 (2009).

[33] L. Wang and X.-F. Han, A light pseudoscalar of 2HDM confronted with muon $g-2$ and experimental constraints, J. High Energy Phys. 05 (2015) 039.

[34] V. Ilisie, New Barr-Zee contributions to $(g-2)_{\mu}$ in twoHiggs-doublet models, J. High Energy Phys. 04 (2015) 077.

[35] T. Abe, R. Sato, and K. Yagyu, Lepton-specific two Higgs doublet model as a solution of muon $g-2$ anomaly, J. High Energy Phys. 07 (2015) 064.

[36] T. Han, S. K. Kang, and J. Sayre, Muon $g-2$ in the aligned two Higgs doublet model, J. High Energy Phys. 02 (2016) 097.

[37] E. J. Chun and J. Kim, Leptonic precision test of leptophilic two-Higgs-doublet model, J. High Energy Phys. 07 (2016) 110.

[38] A. Cherchiglia, P. Kneschke, D. Stöckinger, and H. Stöckinger-Kim, The muon magnetic moment in the 2HDM: Complete two-loop result, J. High Energy Phys. 01 (2017) 007.

[39] A. Cherchiglia, D. Stöckinger, and H. Stöckinger-Kim, Muon $g-2$ in the 2HDM: Maximum results and detailed phenomenology, Phys. Rev. D 98, 035001 (2018).

[40] L. Wang, J. M. Yang, M. Zhang, and Y. Zhang, Revisiting lepton-specific 2HDM in light of muon $g-2$ anomaly, Phys. Lett. B 788, 519 (2019).

[41] E. J. Chun, Z. Kang, M. Takeuchi, and Y.-L. S. Tsai, LHC $\tau$-rich tests of lepton-specific 2HDM for $\left((g-2)_{\mu}\right)$, J. High Energy Phys. 11 (2015) 099.

[42] D. Atwood, L. Reina, and A. Soni, Phenomenology of two Higgs doublet models with flavor changing neutral currents, Phys. Rev. D 55, 3156 (1997).

[43] R. Diaz, R. Martinez, and J. A. Rodriguez, Lepton flavor violation in the two Higgs doublet model type III, Phys. Rev. D 63, 095007 (2001).

[44] Z.-j. Xiao and L. Guo, B0 anti-B0 mixing and B $\rightarrow X(s)$ gamma decay in the third type 2HDM: Effects of NLO QCD contributions, Phys. Rev. D 69, 014002 (2004).

[45] Y. Omura, E. Senaha, and K. Tobe, $\tau$ - and $\mu$-physics in a general two Higgs doublet model with $\mu-\tau$ flavor violation, Phys. Rev. D 94, 055019 (2016).

[46] S. Davidson, $\mu \rightarrow e \gamma$ in the 2HDM: An exercise in EFT, Eur. Phys. J. C 76, 258 (2016).

[47] A. Arhrib, R. Benbrik, C. H. Chen, J. K. Parry, L. Rahili, S. Semlali et al., $R_{K^{(*)}}$ anomaly in type-III 2HDM, arXiv: 1710.05898 .

[48] R. A. Diaz, R. Martinez, and J. A. Rodriguez, Bounds for lepton flavor violation and the pseudoscalar Higgs boson in the general two Higgs doublet model using the $g-2$ muon factor, Phys. Rev. D 64, 033004 (2001).

[49] Y. Omura, E. Senaha, and K. Tobe, Lepton-flavor-violating Higgs decay $h \rightarrow \mu \tau$ and muon anomalous magnetic moment in a general two Higgs doublet model, J. High Energy Phys. 05 (2015) 028. 
[50] G. C. Branco, P. M. Ferreira, L. Lavoura, M. N. Rebelo, M. Sher, and J. P. Silva, Theory and phenomenology of twoHiggs-doublet models, Phys. Rep. 516, 1 (2012).

[51] A. Crivellin, J. Heeck, and P. Stoffer, A Perturbed LeptonSpecific Two-Higgs-Doublet Model Facing Experimental Hints for Physics Beyond the Standard Model, Phys. Rev. Lett. 116, 081801 (2016).

[52] T. P. Cheng and M. Sher, Mass matrix ansatz and flavor nonconservation in models with multiple Higgs doublets, Phys. Rev. D 35, 3484 (1987).

[53] M. Davier, A. Hoecker, B. Malaescu, and Z. Zhang, Reevaluation of the hadronic contributions to the muon $g-2$ and to alpha(MZ), Eur. Phys. J. C 71 (2011) 1515.

[54] K. Hagiwara, R. Liao, A. D. Martin, D. Nomura, and T. Teubner, $(g-2)_{\mu}$ and $\alpha\left(M_{Z}^{2}\right)$ re-evaluated using new precise data, J. Phys. G 38, 085003 (2011).

[55] M. Davier, A. Hoecker, B. Malaescu, and Z. Zhang, Reevaluation of the hadronic vacuum polarisation contributions to the Standard Model predictions of the muon $g-2$ and $\alpha\left(m_{Z}^{2}\right)$ using newest hadronic cross-section data, Eur. Phys. J. C 77, 827 (2017).

[56] M. Davier, A. Hoecker, B. Malaescu, and Z. Zhang, A new evaluation of the hadronic vacuum polarisation contributions to the muon anomalous magnetic moment and to $\boldsymbol{\alpha}\left(\mathbf{m}_{\mathbf{Z}}^{\mathbf{2}}\right)$, Eur. Phys. J. C 80, 241 (2020); Erratum, Eur. Phys. J. C 80, 410 (2020).

[57] T. Aoyama et al., The anomalous magnetic moment of the muon in the Standard Model, Phys. Rep. 887, 1 (2020).

[58] A. Keshavarzi, D. Nomura, and T. Teubner, Muon $g-2$ and $\alpha\left(M_{Z}^{2}\right)$ : A new data-based analysis, Phys. Rev. D 97, 114025 (2018).

[59] G. Colangelo, M. Hoferichter, and P. Stoffer, Two-pion contribution to hadronic vacuum polarization, J. High Energy Phys. 02 (2019) 006.

[60] M. Hoferichter, B.-L. Hoid, and B. Kubis, Three-pion contribution to hadronic vacuum polarization, J. High Energy Phys. 08 (2019) 137.

[61] A. Keshavarzi, D. Nomura, and T. Teubner, $g-2$ of charged leptons, $\alpha\left(M_{Z}^{2}\right)$, and the hyperfine splitting of muonium, Phys. Rev. D 101, 014029 (2020).

[62] A. Kurz, T. Liu, P. Marquard, and M. Steinhauser, Hadronic contribution to the muon anomalous magnetic moment to next-to-next-to-leading order, Phys. Lett. B 734, 144 (2014).

[63] K. Melnikov and A. Vainshtein, Hadronic light-by-light scattering contribution to the muon anomalous magnetic moment revisited, Phys. Rev. D 70, 113006 (2004).

[64] P. Masjuan and P. Sanchez-Puertas, Pseudoscalar-pole contribution to the $\left(g_{\mu}-2\right)$ : A rational approach, Phys. Rev. D 95, 054026 (2017).

[65] G. Colangelo, M. Hoferichter, M. Procura, and P. Stoffer, Dispersion relation for hadronic light-by-light scattering: Two-pion contributions, J. High Energy Phys. 04 (2017) 161.

[66] M. Hoferichter, B.-L. Hoid, B. Kubis, S. Leupold, and S. P. Schneider, Dispersion relation for hadronic light-by-light scattering: Pion pole, J. High Energy Phys. 10 (2018) 141.

[67] A. Gérardin, H. B. Meyer, and A. Nyffeler, Lattice calculation of the pion transition form factor with $N_{f}=2+1$ Wilson quarks, Phys. Rev. D 100, 034520 (2019).
[68] J. Bijnens, N. Hermansson-Truedsson, and A. RodríguezSánchez, Short-distance constraints for the HLbL contribution to the muon anomalous magnetic moment, Phys. Lett. B 798, 134994 (2019).

[69] G. Colangelo, F. Hagelstein, M. Hoferichter, L. Laub, and P. Stoffer, Longitudinal short-distance constraints for the hadronic light-by-light contribution to $(g-2)_{\mu}$ with large$N_{c}$ Regge models, J. High Energy Phys. 03 (2020) 101.

[70] G. Colangelo, M. Hoferichter, A. Nyffeler, M. Passera, and P. Stoffer, Remarks on higher-order hadronic corrections to the muon $g-2$, Phys. Lett. B 735, 90 (2014).

[71] T. Blum, N. Christ, M. Hayakawa, T. Izubuchi, L. Jin, C. Jung, and C. Lehner, Hadronic Light-by-Light Scattering Contribution to the Muon Anomalous Magnetic Moment from Lattice QCD, Phys. Rev. Lett. 124, 132002 (2020).

[72] T. Aoyama, M. Hayakawa, T. Kinoshita, and M. Nio, Complete Tenth-Order QED Contribution to the Muon $g-2$, Phys. Rev. Lett. 109, 111808 (2012).

[73] A. Czarnecki and W. J. Marciano, Refinements in electroweak contributions to the muon anomalous magnetic moment, Phys. Rev. D 67, 073006 (2003).

[74] T. Aoyama, T. Kinoshita, and M. Nio, Theory of the anomalous magnetic moment of the electron, Atoms 7 (2019) 28.

[75] C. Gnendiger, D. Stöckinger, and H. Stöckinger-Kim, The electroweak contributions to $(g-2)_{\mu}$ after the Higgs boson mass measurement, Phys. Rev. D 88, 053005 (2013).

[76] G. W. Bennett et al. (Muon g-2 Collaboration), Final report of the muon E821 anomalous magnetic moment measurement at BNL, Phys. Rev. D 73, 072003 (2006).

[77] F. S. Queiroz and W. Shepherd, New physics contributions to the muon anomalous magnetic moment: A numerical code, Phys. Rev. D 89, 095024 (2014).

[78] A. Crivellin, M. Hoferichter, and M. Procura, Improved predictions for $\mu \rightarrow e$ conversion in nuclei and Higgsinduced lepton flavor violation, Phys. Rev. D 89, 093024 (2014).

[79] R. H. Bernstein and P. S. Cooper, Charged lepton flavor violation: An experimenter's guide, Phys. Rep.532, 27 (2013).

[80] J. F. Gunion and H. E. Haber, The $C P$ conserving two Higgs doublet model: The approach to the decoupling limit, Phys. Rev. D 67, 075019 (2003).

[81] N. G. Deshpande and E. Ma, Pattern of symmetry breaking with two Higgs doublets, Phys. Rev. D 18, 2574 (1978).

[82] A. M. Sirunyan et al. (CMS Collaboration), Search for a light pseudoscalar Higgs boson in the boosted $\mu \mu \tau \tau$ final state in proton-proton collisions at $\sqrt{s}=13 \mathrm{TeV}$, J. High Energy Phys. 08 (2020) 139.

[83] J. Bernon, J. F. Gunion, Y. Jiang, and S. Kraml, Light Higgs bosons in two-Higgs-doublet models, Phys. Rev. D 91, 075019 (2015).

[84] L. Lavoura and L.-F. Li, Making the small oblique parameters large, Phys. Rev. D 49, 1409 (1994).

[85] M. Baak, M. Goebel, J. Haller, A. Hoecker, D. Kennedy, R. Kogler, K. Mönig, M. Schott, and J. Stelzer, The electroweak fit of the standard model after the discovery of a new boson at the LHC, Eur. Phys. J. C 72, 2205 (2012).

[86] M. Baak (Gfitter Collaboration), Review of electroweak fits of the SM and beyond, after the Higgs discovery-with Gfitter, Proc. Sci., EPS-HEP2013 (2013) 203. 
[87] J. Haller, A. Hoecker, R. Kogler, K. Mönig, T. Peiffer, and J. Stelzer, Update of the global electroweak fit and constraints on two-Higgs-doublet models, Eur. Phys. J. C 78, 675 (2018).

[88] A. Crivellin, A. Kokulu, and C. Greub, Flavor-phenomenology of two-Higgs-doublet models with generic Yukawa structure, Phys. Rev. D 87, 094031 (2013).

[89] A. Arbey, F. Mahmoudi, O. Stal, and T. Stefaniak, Status of the charged Higgs boson in two Higgs doublet models, Eur. Phys. J. C 78, 182 (2018).

[90] M. Hussain, M. Usman, M. A. Paracha, and M. J. Aslam, Constraints on Two Higgs Doublet Model Parameters in the light of rare $B$-decays, Phys. Rev. D 95, 075009 (2017).

[91] T. Enomoto and R. Watanabe, Flavor constraints on the two Higgs doublet models of $\mathrm{Z}_{2}$ symmetric and aligned types, J. High Energy Phys. 05 (2016) 002.

[92] R. Alonso, B. Grinstein, and J. Martin Camalich, Lifetime of $B_{c}^{-}$Constrains Explanations for Anomalies in $B \rightarrow D^{(*)} \tau \nu$, Phys. Rev. Lett. 118, 081802 (2017).

[93] C. Patrignani et al. (Particle Data Group Collaboration), Review of Particle Physics, Chin. Phys. C 40, 100001 (2016).

[94] G. Abbiendi et al. (ALEPH, DELPHI, L3, OPAL, LEP Collaborations), Search for charged Higgs bosons: Combined results using LEP data, Eur. Phys. J. C 73, 2463 (2013).

[95] G. Aad et al. (ATLAS Collaboration), Search for charged Higgs bosons decaying via $H^{ \pm} \rightarrow \tau^{ \pm} \nu$ in fully hadronic final states using $p p$ collision data at $\sqrt{s}=8 \mathrm{TeV}$ with the ATLAS detector, J. High Energy Phys. 03 (2015) 088.

[96] V. Khachatryan et al. (CMS Collaboration), Search for a charged Higgs boson in pp collisions at $\sqrt{s}=8 \mathrm{TeV}$, J. High Energy Phys. 11 (2015) 018.

[97] G. Aad et al. (ATLAS Collaboration), Search for a light charged Higgs boson in the decay channel $H^{+} \rightarrow c \bar{s}$ in $t \bar{t}$ events using pp collisions at $\sqrt{s}=7 \mathrm{TeV}$ with the ATLAS detector, Eur. Phys. J. C 73, 2465 (2013).

[98] V. Khachatryan et al. (CMS Collaboration), Search for a light charged Higgs boson decaying to $\mathrm{cs}$ in $\mathrm{pp}$ collisions at $\sqrt{s}=8 \mathrm{TeV}$, J. High Energy Phys. 12 (2015) 178.

[99] M. Aaboud et al. (ATLAS Collaboration), Search for charged Higgs bosons produced in association with a top quark and decaying via $H^{ \pm} \rightarrow \tau \nu$ using $p p$ collision data recorded at $\sqrt{s}=13 \mathrm{TeV}$ by the ATLAS detector, Phys. Lett. B 759, 555 (2016).

[100] T.A. Collaboration (ATLAS Collaboration), Search for charged Higgs bosons in the $\tau+$ jets final state using $14.7 \mathrm{fb}^{-1}$ of pp collision data recorded at of pp collision data recorded at $\sqrt{s}=13 \mathrm{TeV}$ with the ATLAS experiment, ATLAS-CONF-2016-088.

[101] CMS Collaboration, Search for charged Higgs bosons with the $\mathrm{H}^{ \pm} \rightarrow \tau^{ \pm} \nu_{\tau}$ decay channel in the fully hadronic final state at $\sqrt{s}=13 \mathrm{TeV}$, CMS-PAS-HIG-16-031.

[102] ATLAS Collaboration, Search for charged Higgs bosons in the $H^{ \pm} \rightarrow t b$ decay channel in $p p$ collisions at $\sqrt{s}=$ $13 \mathrm{TeV}$ using the ATLAS detector, ATLAS-CONF-2016089.

[103] V. Khachatryan et al. (CMS Collaboration), Search for a low-mass pseudoscalar Higgs boson produced in association with a $b \bar{b}$ pair in $p p$ collisions at $\sqrt{s}=8$ TeV, Phys. Lett. B 758, 296 (2016).

[104] A. M. Sirunyan et al. (CMS Collaboration), Search for a low-mass $\tau^{+} \tau^{-}$resonance in association with a bottom quark in proton-proton collisions at $\sqrt{s}=13 \mathrm{TeV}$, J. High Energy Phys. 05 (2019) 210.

[105] V. Khachatryan et al. (CMS Collaboration), Search for neutral MSSM Higgs bosons decaying into a pair of bottom quarks, J. High Energy Phys. 11 (2015) 071.

[106] CMS Collaboration, Search for a narrow heavy decaying to bottom quark pairs in the $13 \mathrm{TeV}$ data sample, CMS-PASHIG-16-025.

[107] V. Khachatryan et al. (CMS Collaboration), Search for neutral resonances decaying into a $\mathrm{Z}$ boson and $\mathrm{a}$ pair of $\mathrm{b}$ jets or $\tau$ leptons, Phys. Lett. B 759, 369 (2016).

[108] CMS Collaboration, Search for $\mathrm{H}$ to $\mathrm{Z}(1 \mathrm{ll})+\mathrm{A}(\mathrm{bb})$ with 2015 data, CMS-PAS-HIG-16-010.

[109] G. Aad et al. (ATLAS Collaboration), Evidence for the Higgs-boson Yukawa coupling to tau leptons with the ATLAS detector, J. High Energy Phys. 04 (2015) 117.

[110] S. Chatrchyan et al. (CMS Collaboration), Evidence for the $125 \mathrm{GeV}$ Higgs boson decaying to a pair of $\tau$ leptons, J. High Energy Phys. 05 (2014) 104.

[111] G. Aad et al. (ATLAS Collaboration), Search for the Standard Model Higgs boson decay to $\mu^{+} \mu^{-}$with the ATLAS detector, Phys. Lett. B 738, 68 (2014).

[112] V. Khachatryan et al. (CMS Collaboration), Search for a standard model-like Higgs boson in the $\mu \mu$ and $e e$ decay channels at the LHC, Phys. Lett. B 744, 184 (2015).

[113] CMS Collaboration, Search for lepton-flavour-violating decays of the Higgs boson to $e \tau$ and $e \mu$ at $\sqrt{s}=8 \mathrm{TeV}$, CMS-PAS-HIG-14-040.

[114] V. Khachatryan et al. (CMS Collaboration), Search for lepton-flavour-violating decays of the Higgs boson, Phys. Lett. B 749, 337 (2015).

[115] A. M. Sirunyan et al. (CMS Collaboration), Search for lepton flavour violating decays of a neutral heavy Higgs boson to $\mu \tau$ and $e \tau$ in proton-proton collisions at $\sqrt{s}=$ 13 TeV, J. High Energy Phys. 03 (2020) 103.

[116] J. Alwall, R. Frederix, S. Frixione, V. Hirschi, F. Maltoni, O. Mattelaer, H.-S. Shao, T. Stelzer, P. Torrielli, and M. Zaro, The automated computation of tree-level and nextto-leading order differential cross sections, and their matching to parton shower simulations, J. High Energy Phys. 07 (2014) 079.

[117] A. Alloul, N. D. Christensen, C. Degrande, C. Duhr, and B. Fuks, FEYNRULES 2.0-A complete toolbox for treelevel phenomenology, Comput. Phys. Commun. 185, 2250 (2014).

[118] R. D. Ball et al. (NNPDF Collaboration), Parton distributions for the LHC Run II, J. High Energy Phys. 04 (2015) 040.

[119] T. Sjostrand, S. Mrenna, and P. Z. Skands, PYTHIA 6.4 physics and manual, J. High Energy Phys. 05 (2006) 026.

[120] J. de Favereau, C. Delaere, P. Demin, A. Giammanco, V. Lemaître, A. Mertens, and M. Selvaggi (DELPHES 3 Collaboration), DELPHES 3, A modular framework for fast simulation of a generic collider experiment, J. High Energy Phys. 02 (2014) 057. 
[121] A. M. Sirunyan et al. (CMS Collaboration), Identification of heavy-flavour jets with the CMS detector in pp collisions at $13 \mathrm{TeV}$, J. Instrum. 13, P05011 (2018).

[122] G. Cowan, K. Cranmer, E. Gross, and O. Vitells, Asymptotic formulae for likelihood-based tests of new physics, Eur. Phys. J. C 71, 1554 (2011); Erratum, Eur. Phys. J. C 73, 2501 (2013).

[123] A. Djouadi and S. Ferrag, PDF uncertainties in Higgs production at hadron colliders, Phys. Lett. B 586, 345 (2004).

[124] A. M. Sirunyan et al. (CMS Collaboration), Measurement of the $t \bar{t}$ production cross section using events with one lepton and at least one jet in pp collisions at $\sqrt{s}=13 \mathrm{TeV}$, J. High Energy Phys. 09 (2017) 051.

[125] S. Catani, L. Cieri, G. Ferrera, D. de Florian, and M. Grazzini, Vector Boson Production at Hadron Colliders: A Fully Exclusive QCD Calculation at NNLO, Phys. Rev. Lett. 103, 082001 (2009).

[126] L. Teodorescu, Artificial neural networks in high-energy physics, in Computing. Proceedings, inverted CERN School of Computing, ICSC2005 and ICSC2006, Geneva, Switzerland, 2005 and 2006 (CERN, Geneva, 2008), pp. 13-22, http://doc.cern.ch/yellowrep/2008/2008-002/ p13.pdf.
[127] K. Hultqvist, R. Jacobsson, and K. E. Johansson, Using a neural network in the search for the Higgs boson, DELPHI-95-61 PHYS 496.

[128] R. D. Field, Y. Kanev, M. Tayebnejad, and P. A. Griffin, Using neural networks to enhance the Higgs boson signal at hadron colliders, Phys. Rev. D 53, 2296 (1996).

[129] N. Bakhet, M. Yu. Khlopov, and T. Hussein, Neural networks search for charged Higgs boson of two doublet Higgs model at the hadrons colliders, arXiv:1507.06547.

[130] A. Dey, J. Lahiri, and B. Mukhopadhyaya, LHC signals of a heavy doublet Higgs as dark matter portal: Cut-based approach and improvement with gradient boosting and neural networks, J. High Energy Phys. 09 (2019) 004.

[131] K. Lasocha, E. Richter-Was, M. Sadowski, and Z. Was, Deep neural network application: Higgs boson $C P$ state mixing angle in $\mathrm{H}$ to tau tau decay and at LHC, arXiv: 2001.00455.

[132] A. Dey, J. Lahiri, and B. Mukhopadhyaya, LHC signals of triplet scalars as dark matter portal: Cut-based approach and improvement with gradient boosting and neural networks, J. High Energy Phys. 06 (2020) 126.

[133] J. R. Hermans, Distributed Keras: Distributed deep learning with apache spark and keras, CERN IT-DB, https:// github.com/cerndb/dist-keras. 